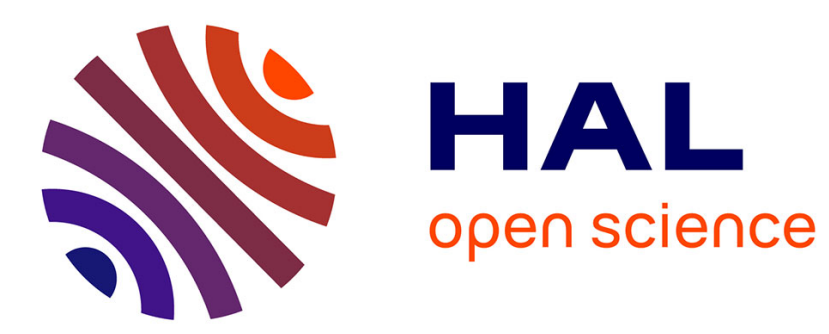

\title{
Thinning and harvesting in stochastic forest models
}

Kurt L. Helmes, Richard H. Stockbridge

\section{To cite this version:}

Kurt L. Helmes, Richard H. Stockbridge. Thinning and harvesting in stochastic forest models. Journal of Economic Dynamics and Control, 2010, 35 (1), pp.25. 10.1016/j.jedc.2010.10.007 . hal-00753039

\section{HAL Id: hal-00753039 \\ https://hal.science/hal-00753039}

Submitted on 17 Nov 2012

HAL is a multi-disciplinary open access archive for the deposit and dissemination of scientific research documents, whether they are published or not. The documents may come from teaching and research institutions in France or abroad, or from public or private research centers.
L'archive ouverte pluridisciplinaire HAL, est destinée au dépôt et à la diffusion de documents scientifiques de niveau recherche, publiés ou non, émanant des établissements d'enseignement et de recherche français ou étrangers, des laboratoires publics ou privés. 


\section{Author's Accepted Manuscript}

Thinning and harvesting in stochastic forest models

Kurt L. Helmes, Richard H. Stockbridge

PII: $\quad$ S0165-1889(10)00235-6

DOI: $\quad$ doi:10.1016/j.jedc.2010.10.007

Reference: $\quad$ DYNCON 2497

To appear in: $\quad$ Journal of Economic Dynamics \& Control

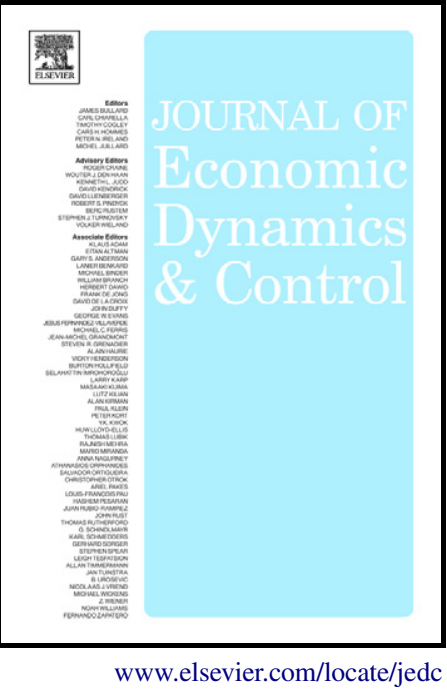

Received date: $\quad 10$ April 2009

Revised date: $\quad 12$ April 2010

Accepted date: 1 June 2010

Cite this article as: Kurt L. Helmes and Richard H. Stockbridge, Thinning and harvesting in stochastic forest models, Journal of Economic Dynamics \& Control, doi:10.1016/j.jedc.2010.10.007

This is a PDF file of an unedited manuscript that has been accepted for publication. As a service to our customers we are providing this early version of the manuscript. The manuscript will undergo copyediting, typesetting, and review of the resulting galley proof before it is published in its final citable form. Please note that during the production process errors may be discovered which could affect the content, and all legal disclaimers that apply to the journal pertain. 


\title{
Thinning and Harvesting in Stochastic Forest Models
}

\author{
Kurt L. Helmes ${ }^{\mathrm{a}, *}$, Richard H. Stockbridge ${ }^{\mathrm{b}, 1, * *}$ \\ ${ }^{a}$ Humboldt-Universität zu Berlin, Berlin, Germany \\ ${ }^{b}$ University of Wisconsin Milwaukee, Milwaukee, WI 53201, USA
}

\begin{abstract}
This paper analyzes a stochastic forest growth model in which the manager is able to first thin the forest to promote better growth before harvesting. Both Wicksell single thinning-and-harvesting cycle and Faustmann on-going rotation problems are considered. The Wicksell problem is analyzed by first restricting the class of decision times to (thinning,harvesting) pairs that bound the growth away from infinity and imbedding the problem in an infinite-dimensional linear program on a space of triplets of measures. These measures capture the thinning and harvesting decisions along with the behavior of the growth process prior to harvest. An auxiliary linear program then leads to a nonlinear optimization problem for which an optimal value and solution are determined. The values of all the problems are be related through a set of inequalities. The solution of the nonlinear problem determines (random) thinning and harvesting times for the single thinningand-harvesting cycle which demonstrate the equality of the values of these various problems. Finally for the Wicksell problem, the unrestricted class of thinning-and-harvest times is shown to give the same value as the restricted class. The Faustmann on-going thinning-and-harvesting rotation problem is reduced to a Wicksell problem which then allows for the characterization of the value as the solution to a different nonlinear optimization problem. The effects of the opportunity to thin the forest are illustrated on a mean-reverting stochastic model.
\end{abstract}

Keywords: stochastic forest models, forest rotation, Wicksell, Faustmann, harvest, thinning, linear programming

JEL: C61, Q23

\section{Introduction}

Consider a stochastic forest model where, without any intervention, the process $X$ is assumed to satisfy

$$
d X(t)=\mu(X(t), Y(t)) d t+\sigma(X(t), Y(t)) d W(t), \quad X(0)=x_{n e w}>0 .
$$

Here $X$ is a process that captures the volume and quality of the forest stand, $x_{n e w}$ is the value of a "new" forest, $\mu$ denotes the mean growth rate, $\sigma$ denotes the volatility of the growth rate and $W$ is a standard Brownian motion process which provides the random fluctuations of the forest size. The process $Y$ is an indicator process that identifies whether the forest is new and densely planted $(Y(t)=1)$ or has been thinned $(Y(t)=2)$; we assume $Y(0)=1$. For our model, we have in mind functions $\mu$ and $\sigma$ such that the process $X$ is always nonnegative and represents a growth process. Allowing dependence on the process $Y$ in these coefficients means that the growth dynamics can differ for dense and thinned forests or even that the process

\footnotetext{
*Corresponding author

** Principal corresponding author

Email addresses: helmes@wiwi.hu-berlin.de (Kurt L. Helmes), stockbri@csd.uwm.edu (Richard H. Stockbridge)

${ }^{1}$ This research has been supported in part by the U.S. National Security Agency under Grant Agreement Number H9823009-1-0002. The United States Government is authorized to reproduce and distribute reprints notwithstanding any copyright notation herein.
} 
$X$ can represent different quantities for the two types of forest. For example, $X$ may represent the volume of fuel wood for a dense forest and the diameter at breast height (dbh) for an average, more valuable tree in the thinned forest. The interventions in the growth of the forest occur when it is thinned and harvested. This paper analyzes both a single thinning-harvesting cycle of Wicksell type and the Faustmann on-going rotation problem for this two-decision model.

For the Wicksell single-cycle problem, the goal of the forest manager is to maximize the expected presentvalue of the net proceeds of the forest product when thinned and harvested. Let $\theta$ denote the time at which the manager thins the forest and let $\eta$ be the harvest time. The size of the forest after thinning is $X(\theta)$ whereas the size of the stand is $X(\theta-)$ at the time the decision to thin is made; in this model, the thinning decision results in an immediate jump to the process $X$. Let $g_{1}$ and $g_{2}$ denote the net profit functions from thinning and harvesting, respectively, and let $\alpha>0$ denote the discount rate. The single-cycle objective is one of selecting times $\theta$ and $\eta$, with $\theta \leq \eta$, so as to maximize

$$
E\left[e^{-\alpha \theta} g_{1}(X(\theta-)) I_{\{\theta<\infty\}}+e^{-\alpha \eta} g_{2}(X(\eta)) I_{\{\eta<\infty\}}\right] .
$$

Let $V^{w}\left(x_{n e w}, 1\right)$ denote the optimal value.

At this point, one must mention the importance of the interpretation of the model for the dense and thinned states. The process $X$ may possibly represent two different quantities in the two states, in which case it may be reasonable for thinning to result in an increase to the process $X ; X(\theta)>X(\theta-)$. When the model is such that $X$ represents the same quantity for both dense and thinned forests, it may be necessary to impose an additional condition on the decision times of the model. For example, if $X$ were to represent the volume of lumber on the stand, then a decision to thin would reduce the size of $X$ and the model would therefore restrict the thinning decision to occur only after the process $X$ exceeds the size of the thinned stand. If $X(\theta)=x_{t h i n}$, in the simplest case, then one would require $\theta>\tau_{x_{t h i n}}$, where $\tau_{x_{t h i n}}$ is the first time at which $X$ achieves level $x_{\text {thin }}$. This paper treats the more general model by allowing $X$ to increase in value when thinning occurs; the results can be easily adapted to include additional restrictions on the decision times.

The Faustmann rotation problem replants trees for the cycle to repeat with the new planting resulting in the forest stand returning to value $x_{n e w}$ so the process $X$ starts from this point following a harvest decision. For $k=1,2,3, \ldots$, let $\theta_{k}$ and $\eta_{k}$ denote the random times at which the forest is thinned and harvested, respectively, for the $k^{\text {th }}$ time. Then for each $k, X\left(\theta_{k}-\right)$ and $X\left(\eta_{k}-\right)$ represent the values of $X$ when the decisions are made to thin and harvest, respectively, while $X\left(\theta_{k}\right)$ and $X\left(\eta_{k}\right)=x_{n e w}$ denote the sizes of $X$ immediately following these interventions. The Faustmann on-going rotation problem is one of maximizing

$$
E\left[\sum_{k=1}^{\infty}\left(e^{-\alpha \theta_{k}} g_{1}\left(X\left(\theta_{k}-\right)\right) I_{\left\{\theta_{k}<\infty\right\}}+e^{-\alpha \eta_{k}} g_{2}\left(X\left(\eta_{k}-\right)\right) I_{\left\{\eta_{k}<\infty\right\}}\right)\right]
$$

Let $V^{f}\left(x_{n e w}, 1\right)$ denote the optimal value for the Faustmann problem.

The mathematical modeling of forest growth with the aim of determining on-going optimal harvesting decisions began with the paper by Faustmann (1849) which considered the case of deterministic growth. Since 1849, a large literature has developed on this topic. The bibliography by Newman (2002) provides a partial but extensive up-to-date (2002) list of references on the economics of forest rotation. We concentrate our comments on some papers using stochastic models. Nordstrøm (1975) modelled the growth process deterministically but introduced randomness with prices that followed a finite-state Markov chain in discrete time. Miller and Voltaire (1983) consider a diffusion process for the tree size and solve the rotation problem. A limitation of this model is that tree sizes will become negative. Clarke and Reed (1989) use a geometric Brownian motion for the forest size to ensure positivity and an age-dependent geometric Brownian motion for the price process. Using optimal stopping methods, the paper analyzes both the Wicksell single-period and Faustmann on-going harvest rotation problems. Willassen (1998) considers a general stochastic differential equation model for the growth process in continuous time and uses impulse control methods to solve the problem. Buongiorno (2001) and others employ Markov decision processes to model the growth process in discrete time; Buongiorno reformulates the problem as a finite-dimensional linear program. Sødal (2002) 
restricts his analysis to decision times that are hitting times of the growth process at fixed levels and uses an intuitive mark-up pricing approach to characterize the value as a nonlinear optimization problem. Additional work on the Wicksell single-period problem for stochastic growth models include papers by Alvarez and Koskela (see, e.g., Alvarez and Koskela (2005)), among others. Penttinen (2006) includes thinning considerations in his models but only in terms of cost, not with the possibility of the growth dynamics improving.

Though our exposition is expressed entirely in terms of forests, their harvest, rotation and thinning, the paper by Miller and Voltaire (1983) discusses how the rotation problem is a paradigm that has many additional economic applications.

The thinning and harvest decisions represent times when the decision maker elects to switch from one diffusion process to the other. Switching problems have also been considered in the literature where from a mathematical point of view such decisions are phrased in terms of optimal stopping, impulse control or more general stochastic control problems. The traditional approach is one of solving the Hamilton-Jacobi-Bellman (HJB) equation or variational or quasi-variational inequalities associated with the given problem (see, e.g., Baldursson and Karatzas (1997), Brekke and Øksendal (1994), Duckworth and Zervos (2001), Lumley and Zervos (2001), Zervos (2002) and references therein, for a short selection of recent publications). This indirect approach seeks the solutions to a family of optimization problems that are parametrized by the initial position of the process. The mathematical challenge is to prove optimality within a large class of policies and to prove the existence of a solution of an HJB-equation or variational inequality under weak conditions and mild regularity assumptions imposed on the data.

The thinning-and-harvest problem is also similar to entry-and-exit problems in economics in which the decision maker determines times at which to enter a market and times at which to exit from the market. This type of problem was introduced by Brennan and Schwartz (1985) and further analyzed by Dixit (1989) and Dixit and Pindyck (1994). These papers, as do some of the ones mentioned in the previous paragraph, only consider a single diffusion process rather than switching diffusions.

This paper approaches the optimal switching problem quite differently by concentrating on the optimization problem corresponding to a single initial value rather than addressing the parametrized family of problems. The stochasticity of the problem is captured by occupation measures that must satisfy an identity (see (7) below) for a large class of test functions. The class is rich enough that one is able to reconstruct the stochastic process, though this paper does not take advantage of this fact; see, for example, (Kurtz and Stockbridge, 1998, 2001) for more details. The measures become the variables for an infinite-dimensional linear program that provides an upper bound on the optimal value. The constraints of this linear program are then relaxed to obtain an auxiliary linear program for which a further upper bound is easily determined. This final upper bound arises as the value of a nonlinear optimization problem in two variables. Using optimal values of these variables allows one to determine sequences of decision times such that the value of the original problem equals the highest upper bound and hence these times are seen to be optimal.

This paper is organized as follows. Section 2 concentrates on the Wicksell single thinning-and-harvesting cycle problem, beginning by placing a restriction on the class of decision times. The restricted stochastic problem is imbedded in an infinite-dimensional linear program in Section 2.1 having variables in a space of triplets of measures. Section 2.2 develops the auxiliary linear program and corresponding nonlinear optimization problem in such a manner that all of the values for the various problems can be related by inequalities. It also characterizes an optimal solution within the class of restricted decision times. The unrestricted problem is shown to have the same value in Section 2.3 and hence the optimal thinning and harvest times for the restricted problem are seen to be optimal for the unrestricted problem as well. Section 3 examines the Faustmann thinning-and-harvesting rotation problem. The strong Markov property is employed in Section 3.1 to reduce the Faustmann problem to a Wicksell single-cycle problem with a slight modification to the harvest payoff function. As a result, the value is characterized as the solution to a different nonlinear optimization problem. Illustrative examples using a mean-reverting growth process are given in Sections 2.4 and 3.2 .

The imbedding of the stochastic problem in an infinite-dimensional linear program can often be sharpened to show equivalence between the formulations. Kurtz and Stockbridge (1998) establishes this equivalence for absolutely continuous stochastic control problems, Cho and Stockbridge (2002) proves this for optimal 
stopping problems in which the processes exclude singular behavior (such as the thinning decisions of this paper) and Helmes and Stockbridge (2007) extends this result to processes having singular behavior. These results are proven with less regularity of the payoff functions, typically only requiring semi-continuity. The linear programming formulation then allows one to employ numerical techniques to approximate the optimal solutions. A variety of such numerical approaches are possible, including approximating the diffusion process by a continuous-time Markov chain, characterizing the measures using their moments and using finiteelements to determine densities for approximating measures. The determination of the occupation measures of an optimal process can then be used to determine other quantities of interest about the process. The model and analysis of this paper do not require the equivalence since the equality of the values is derived from the exact analysis.

The linear programming approach extends, for example, to the type of problem considered by Lumley and Zervos (2001) in which in addition to the times at which to begin production or shut it down, the decision maker needs to select the level of production as well. For such problems, the occupation measures would include a component related to the level of production.

\subsection{Detailed Formulation}

We begin with a precise formulation of the growth model with thinning. Assume the coefficients $\mu$ and $\sigma$ are continuous and are such that the process $X_{y}, y=1,2$, is a weak solution of the stochastic differential equation

$$
d X_{y}(t)=\mu\left(X_{y}(t), y\right) d t+\sigma\left(X_{y}(t), y\right) d W(t), \quad X_{y}(0)=0
$$

(see Ethier and Kurtz, 1986, Section 5.3, pg. 291, for details) and that these solutions are unique in distribution. Let $A_{y}$ denote the generator of the process $X_{y}$ given by $A_{y} f(x)=\mu(x, y) f^{\prime}(x)+\left(\sigma^{2}(x, y) / 2\right) f^{\prime \prime}(x)$. The uniqueness in distribution then implies that the martingale problems for $A_{y}$ are well-posed and hence that each $X_{y}$ is a strong Markov process (see Ethier and Kurtz, 1986, Theorem 4.4.2, pg. 184). We wish to take advantage of the strong Markov property to piece together the solutions $X_{1}$ and $X_{2}$ to form a weak solution $(X, Y)$ of $(1)$ at all times other than thinning and harvest times. Let $\left\{\mathcal{F}_{t}\right\}$ denote a common filtration with respect to which $X_{1}$ anf $X_{2}$ are weak solutions of (4) for their respective values of $y$.

For the Wicksell problem, recall $\theta$ and $\eta$ denote the thinning and harvesting times, respectively; $\theta$ and $\eta$ are required to be $\left\{\mathcal{F}_{t}\right\}$-stopping times with $\theta \leq \eta$. For a given pair $(\theta, \eta)$, define the paired process $(X, Y)$ as follows. The initial values are $X(0)=x_{n e w}$ and $Y(0)=1$. For $0<t<\theta, X(t)=x_{n e w}+X_{1}(t)$ and $Y(t)=1$. At time $\theta, X$ jumps from $X(\theta-)$ to $X(\theta)$, where $X(\theta)$ has distribution $\pi$ on a bounded interval $\left[x_{\text {min }}, x_{\text {max }}\right]$, with $x_{\text {min }}>0$, and $Y(\theta)=2$. The thinned level of the forest $X(\theta)$ is assumed to be independent of the thinning time $\theta$. For $t>\theta, X(t)=X(\theta)+X_{2}(t-\theta)$. Since the Wicksell payoff ends at the harvest time, we may stop the process at $\eta$ and specify $X(\eta)=X(\eta-)$.

The Faustmann model is quite similar, but with a couple of important changes. The thinning and harvest times are $\left\{\theta_{k}\right\}$ and $\left\{\eta_{k}\right\}$, respectively. Define $\eta_{0}=0$. These decision times must be $\left\{\mathcal{F}_{t}\right\}$-stopping times that satisfy $\eta_{k-1} \leq \theta_{k} \leq \eta_{k}$, for each $k \in \mathbb{N}$. Again, for each $k \in \mathbb{N}$, let $\left\{X_{y}^{(k)}\right\}$ be a sequence of independent processes satisfying $(4), y=1,2$. At each harvest time $\eta_{k-1}$, set $\left(X\left(\eta_{k-1}\right), Y\left(\eta_{k-1}\right)\right)=\left(x_{n e w}, 1\right)$ and for $\eta_{k-1} \leq t<\theta_{k}$, set $X(t)=x_{n e w}+X_{1}^{(k)}\left(t-\eta_{k-1}\right)$ and $Y(t)=1$. At each thinning time $\theta_{k}, X\left(\theta_{k}\right)$ is chosen from $\left[x_{\min }, x_{\max }\right]$ according to $\pi$, independently of $\left\{\theta_{k}\right\}$ and $\left\{\eta_{k}\right\}$ and $Y\left(\theta_{k}\right)=2$. Then for $\theta_{k} \leq t<\eta_{k}$, define $X(t)=X\left(\theta_{k}\right)+X_{2}^{(k)}\left(t-\theta_{k}\right)$ and $Y(t)=2$. Notice that at the times $\theta_{k}$, the forest is thinned to a level $X\left(\theta_{k}\right)$ within some range and this is assumed to happen instantaneously, and at the times $\eta_{k}$, the forest is instantaneously harvested and replanted so $X\left(\eta_{k}\right)=x_{n e w}$, with a corresponding decrease $X\left(\eta_{k}\right)-X\left(\eta_{k}-\right)$ to the process. Observe for each cycle, the process has "poorer growth" dynamics (4) with $y=1$ between $\eta_{k-1}$ and $\theta_{k}$ and "better growth" dynamics (4) with $y=2$ for times between $\theta_{k}$ and $\eta_{k}$.

Let $\mathcal{A}$ denote the set of pairs of admissible decision rules $(\theta, \eta)$ for the Wicksell problem and, with a slight abuse of notation, let $\mathcal{A}$ also denote the sequence of pairs $\left\{\left(\theta_{k}, \eta_{k}\right)\right\}$ for the Faustmann problem.

We place additional restrictions on the coefficients $\mu$ and $\sigma$ through the behavior of the process at the boundaries. Assume $\infty$ is a natural boundary point (see (Borodin and Salminen, 2002, II.1.6, pp. 14, 15) or (Itô and McKean, Jr., 1974, pp. 128-131)) so that the forest does not grow without bounds in a finite time. Also assume that 0 is either a natural, entrance-not-exit or exit-not-entrance boundary point. In the former 
two cases, the forest will never die out in finite time ( that once the forest fails, it never recovers on its own. For models in which 0 is an exit boundary point, the objective function would be non-positive for those decision times $\theta$ and $\eta$ that exceed the hitting time $\zeta$ of $X$ at 0 .

We note that $\left\{\mathcal{F}_{t}\right\}$ is the filtration associated with the weak solution to (1) so it may contain more information than that arising from the observations of the process $X$. Since the stopping times in $\mathcal{A}$ are $\left\{\mathcal{F}_{t}\right\}$-stopping times, these may in principle be determined using information contained in $\left\{\mathcal{F}_{t}\right\}$ that is not generated by $X$. Our results nevertheless show that optimal decision rules exist within the subclass of hitting times of the process.

Before addressing the reward structure for the class of problems under consideration, it is important to describe some additional structure to the problem. The change in dynamics occurs only when the thinning and harvesting decisions are made and hence $Y$ changes only at these decision times. As a result, except at the thinning and harvest decision times, the generator of the pair $(X, Y)$ is

$$
A f(x, y)=\mu(x, y) \frac{\partial f}{\partial x}(x, y)+\frac{\sigma^{2}(x, y)}{2} \frac{\partial^{2} f}{\partial x^{2}}(x, y)
$$

operating on functions $f: \mathbb{R}^{+} \times\{1,2\} \rightarrow \mathbb{R}$ that are twice-continuously differentiable in $x$ for each $y$. Due to the discounting at rate $\alpha$, the eigenvalue problem

$$
A f=\alpha f
$$

plays a central role in the analysis. Under the conditions assumed in this paper, for each $y \in\{1,2\}$, $A f(\cdot, y)=\alpha f(\cdot, y)$ has a nonnegative, strictly increasing solution $\psi_{y}$ (unique up to a positive multiplicative constant) and furthermore $\psi_{y}(0+) \geq 0$ and $\lim _{x \rightarrow \infty} \psi_{y}(x)=\infty$ (see Borodin and Salminen, 2002, II.1.10, pp. 18, 19). The specification of the model and the strictly increasing functions $\psi_{1}$ and $\psi_{2}$ are used to determine restrictions on the payoff functions $g_{1}$ and $g_{2}$ allowed in this paper.

Condition 1. The payoff functions $g_{1}$ and $g_{2}$ are assumed to satisfy the following:

(a) $g_{1}$ and $g_{2}$ are continuous and non-decreasing on $[0, \infty)$;

(b) $g_{1}\left(x_{\text {new }}\right)<0$ and $g_{2}\left(x_{\max }\right) \leq 0$;

(c) there exists some $\bar{x}<\infty$ such that $g_{2}(\bar{x})>0$ and $g_{1}(\bar{x})+\frac{\psi_{2}\left(x_{\min }\right)}{\psi_{2}(\bar{x})} \cdot g_{2}(\bar{x})>0$; and

(d) for $y=1,2, \lim _{x \rightarrow \infty} \frac{g_{y}(x)}{\psi_{y}(x)}=0$

Condition 1(a) implies that a higher value of $X$ yields a larger profit, whereas Condition $1(\mathrm{~b})$ indicates there is non-zero cost to immediately thin a replanted forest and no profit from immediately harvesting the thinned forest. Condition 1(c) means that a sufficiently large value of $X$ will return a positive profit for both harvesting and for thinning followed by harvesting; should this not be the case, then the optimal times $\theta$ and $\eta$ would be infinite so that no cost is incurred. Finally, Condition 1(d) places a restriction on how quickly the reward rate can grow relative to the size of $X$. This condition will be needed in order to eliminate decisions to thin or harvest at arbitrarily large values from being near-optimal decisions.

\section{Wicksell Single Thinning-and-Harvesting Cycle}

This section analyzes the single-cycle problem of deciding when to thin and when to harvest a forest so as to maximize $(2)$ over $(\theta, \eta) \in \mathcal{A}$ with $\theta \leq \eta$. The examination of this problem, however, begins by restricting the decisions to a smaller collection $\mathcal{A}_{1}$ of $\left\{\mathcal{F}_{t}\right\}$-stopping times. The restricted problem is imbedded in an infinite-dimensional linear program from which an auxiliary linear program is derived and a finite-dimensional nonlinear optimization problem arises. An optimal pair $\left(\theta^{*}, \eta^{*}\right)$ of decision times is determined using an optimal solution to the nonlinear problem. Finally, the optimal solution for the 
restricted problem is shown to be optimal for the unrestricted problem and these results are illustrated by an example.

Let $\mathcal{A}_{1}$ denote the collection of pairs of $\left\{\mathcal{F}_{t}\right\}$-stopping times $(\theta, \eta)$, with $\theta \leq \eta$, for which there exists some $K<\infty$ such that $P(X(t) \leq K, 0 \leq t \leq \eta)=1$. For such stopping times, the process $X$ is bounded away from $\infty$. Note that $K$ may differ for different $(\theta, \eta) \in \mathcal{A}_{1}$. Let $V_{r}^{w}\left(x_{n e w}, 1\right)$ denote the optimal value over the restricted collection of pairs of stopping times.

\subsection{Linear Program Imbedding}

Select $(\theta, \eta) \in \mathcal{A}_{1}$ arbitrarily and define the process $\lambda_{\theta}$ by $\lambda_{\theta}(t)=I_{[0, t]}(\theta)$. Notice that $\lambda_{\theta}$ starts at 0 and jumps to 1 at the random time $\theta$ after which it remains at 1 . Let $\mathcal{D}=C_{c}^{2}\left(\mathbb{R}^{+} \times\{1,2\}\right)$, the space of twice-continuously differentiable functions having compact support. For $f \in \mathcal{D}$, an application of Itô's formula results in

$$
\begin{aligned}
e^{-\alpha t} f(X(t), Y(t))= & f\left(x_{n e w}, 1\right)+\int_{0}^{t} e^{-\alpha s}[A f-\alpha f](X(s), Y(s)) d s \\
& +\int_{0}^{t} e^{-\alpha s} \int[f(x, 2)-f(X(s-), Y(s-))] \pi(d x) d \lambda_{\theta}(s) \\
& +\int_{0}^{t} e^{-\alpha s} \sigma(X(s), Y(s)) \frac{\partial f}{\partial x}(X(s), Y(s)) d W(s) .
\end{aligned}
$$

The conditions on $f$ imply that the stochastic integral is a martingale so the optional sampling theorem (see Ethier and Kurtz, 1986, Theorem 2.2.13) establishes that

$$
\begin{aligned}
e^{-\alpha(t \wedge \eta)} f(X(t \wedge \eta), Y(t \wedge \eta)) & -f\left(x_{n e w}, 1\right)-\int_{0}^{t \wedge \eta} e^{-\alpha s}[A f-\alpha f](X(s), Y(s)) d s \\
& -\int_{0}^{t \wedge \eta} e^{-\alpha s} \int[f(x, 2)-f(X(s-), Y(s-))] \pi(d x) d \lambda_{\theta}(s) \\
& =f\left(x_{n e w}, 1\right)+\int_{0}^{t \wedge \eta} e^{-\alpha s} \sigma(X(s), Y(s)) \frac{\partial f}{\partial x}(X(s), Y(s)) d W(s)
\end{aligned}
$$

and hence taking expectations and letting $t \rightarrow \infty$ results in Dynkin's formula:

$$
\begin{aligned}
E\left[e^{-\alpha \eta} f(X(\eta), Y(\eta)) I_{\{\eta<\infty\}}\right] & -E\left[\int_{0}^{\eta} e^{-\alpha s}[A f-\alpha f](X(s), Y(s)) d s\right] \\
& -E\left[\int_{0}^{\eta} e^{-\alpha s} \int[f(x, 2)-f(X(s-), Y(s-))] \pi(d x) d \lambda_{\theta}(s)\right] \\
& =f\left(x_{n e w}, 1\right) .
\end{aligned}
$$

Now define three measures as follows: let $\nu_{\eta}$ denote the discounted distribution of $(X(\eta), Y(\eta))$; let $\nu_{\theta}$ denote the discounted distribution of $(X(\theta-), Y(\theta-))$; and define the expected discounted occupation measure $\nu_{0}$ so that for each $G_{1} \in \mathcal{B}\left(\mathbb{R}^{+}\right)$and $y \in\{1,2\}$

$$
\nu_{0}\left(G_{1} \times\{y\}\right)=E\left[\int_{0}^{\eta} e^{-\alpha s} I_{G_{1} \times\{y\}}(X(s), Y(s)) d s\right] .
$$

Observe that $Y(\eta)=2$ and $Y(\theta-)=1$ so $\nu_{\eta}$ and $\nu_{\theta}$ can be (and, in the sequel, are) measures on $\mathbb{R}^{+}$. Notice also that the total masses of $\nu_{\eta}$ and $\nu_{\theta}$ are bounded above by 1 and the total mass of $\nu_{0}$ is bounded above by $1 / \alpha$. Finally observe that the discounting implies that the sets $\{\theta=\infty\}$ and $\{\eta=\infty\}$ contribute no mass to $\nu_{\theta}$ and $\nu_{\eta}$. 
For a function $h$ on $\left[x_{\min }, x_{\max }\right]$, define $\langle h, \pi\rangle=\int h(x) \pi(d x)$. Using the definitions of the measures $\nu_{\theta}$, $\nu_{\eta}$ and $\nu_{0}$ along with the fact that $\pi$ is a probability measure, (6) can be rewritten as

$$
\begin{aligned}
\int f(x, 2) \nu_{\eta}(d x) & -\int[A f-\alpha f](x, y) \nu_{0}(d x \times d y) \\
& -\int[\langle f(\cdot, 2), \pi\rangle-f(x, 1)] \nu_{\theta}(d x)=f\left(x_{n e w}, 1\right)
\end{aligned}
$$

and this identity holds for all $f \in \mathcal{D}$ and $(\theta, \eta) \in \mathcal{A}_{1}$. (The identity (7) also holds for all $(\theta, \eta) \in \mathcal{A}$ but the ensuing argument requires the stopping times to be in $\mathcal{A}_{1}$.) These measures can be used to evaluate the expected payoff (2) resulting in

$$
\int g_{1}(x) \nu_{\theta}(d x)+\int g_{2}(x) \nu_{\eta}(d x)
$$

Since for stopping times $(\theta, \eta) \in \mathcal{A}_{1}$, the measures $\nu_{\eta}, \nu_{\theta}$ and $\nu_{0}$ satisfy $(7)$ and the corresponding value of (2) is given by (8), the stochastic decision problem on when to thin and harvest over the restricted class of decision rules is imbedded in the linear program

$$
\begin{cases}\text { Maximize } & \int g_{1} d \nu_{\theta}+\int g_{2} d \nu_{\eta} \\ \text { Subject to } & \int f(x, 2) \nu_{\eta}(d x)-\int[A f-\alpha f](x, y) \nu_{0}(d x \times d y) \\ & -\int[\langle f(\cdot, 2), \pi\rangle-f(x, 1)] \nu_{\theta}(d x)=f\left(x_{n e w}, 1\right), \quad \forall f \in \mathcal{D} \\ & \int 1 d \nu_{\theta} \leq 1 \\ & \int 1 d \nu_{\eta} \leq 1 \\ & \int 1 d \nu_{0} \leq 1 / \alpha \\ & \nu_{\theta}, \nu_{\eta}, \nu_{0} \text { measures. }\end{cases}
$$

Let $V_{l p}^{w}\left(x_{n e w}, 1\right)$ denote the value of $(9)$. The above argument immediately implies the following comparison of values.

Theorem 1. $V_{r}^{w}\left(x_{n e w}, 1\right) \leq V_{l p}^{w}\left(x_{n e w}, 1\right)$.

\subsection{Auxiliary Linear Program and Nonlinear Optimization}

The goal now is to simplify the linear program into an auxiliary linear program in a manner that becomes more tractable and for which the values can be easily related. Choose any $(\theta, \eta) \in \mathcal{A}_{1}$ and let $K$ denote the bound corresponding to this pair. Recall, for each $y \in\{1,2\}, \psi_{y}$ is a strictly increasing solution to $A f(\cdot, y)=\alpha f(\cdot, y)$. Let $f(x, y)=a_{1} \psi_{1}(x) I_{\{1\}}(y)+a_{2} \psi_{2}(x) I_{\{2\}}(y)$ for some $a_{1}, a_{2} \in \mathbb{R}$. This function does not have compact support so cannot be immediately used in (7). Let $\xi: \mathbb{R}^{+} \rightarrow \mathbb{R}^{+}$be a mollifying function satisfying $\xi(x)=x$ for $x \leq K$ and $\xi \in C_{c}^{2}\left(\mathbb{R}^{+}\right)$. Considering the function $\tilde{f}(x, y)=\xi(x) \cdot f(x, y)$, we see that $\tilde{f} \in \mathcal{D}$ so can be used in (7). Moreover, for $x \leq K, \frac{\partial \tilde{f}}{\partial x}=\frac{\partial f}{\partial x}$ and $\frac{\partial^{2} \tilde{f}}{\partial x^{2}}=\frac{\partial^{2} f}{\partial x^{2}}$. Since $X(t) \leq K$ a.s. for $t \leq \eta$, it immediately follows that $[A \tilde{f}-\alpha \tilde{f}](X(t), Y(t))=0$ for all $t \leq \eta$ and therefore

$$
\int a_{2} \psi_{2}(x) \nu_{\eta}(d x)-\int\left[a_{2}\left\langle\psi_{2}, \pi\right\rangle-a_{1} \psi_{1}(x)\right] \nu_{\theta}(d x)=a_{1} \psi_{1}\left(x_{n e w}\right) .
$$

Thus when restricting the pairs of decision times $(\theta, \eta)$ to the subcollection $\mathcal{A}_{1}$, the identity $(7)$ extends to the function $f=a_{1} \psi_{1} I_{\{1\}}+a_{2} \psi_{s} I_{\{2\}}$, for any $a_{1}, a_{2} \in \mathbb{R}$.

We now define an auxiliary linear program by replacing the infinite collection of constraints in (9) by two constraints derived from (10) in which the first constraint selects $a_{1}=a_{2}=1$, while the second constraint takes $a_{1}=0$ and $a_{2}=1$. Further relax the conditions by removing the total mass constraints on $\nu_{\theta}, \nu_{\eta}$ and $\nu_{0}$, thus eliminating $\nu_{0}$ from the linear program. The auxiliary linear program is

$$
\begin{cases}\text { Maximize } & \int g_{1}(x) \nu_{\theta}(d x)+\int g_{2}(x) \nu_{\eta}(d x) \\ \text { Subject to } & \int\left[\psi_{1}(x)-\left\langle\psi_{2}, \pi\right\rangle\right] \nu_{\theta}(d x)+\int \psi_{2}(x) \nu_{\eta}(d x)=\psi_{1}\left(x_{n e w}\right), \\ & \int\left\langle\psi_{2}, \pi\right\rangle \nu_{\theta}(d x)-\int \psi_{2}(x) \nu_{\eta}(d x)=0 \\ & \nu_{\eta}, \nu_{\theta} \text { measures. }\end{cases}
$$


Inherent in the formulation (11) is the feasibility requirement on the measures that the function $\psi_{1}$ is integrable with respect to $\nu_{\theta}$ and $\psi_{2}$ is integrable with respect to $\nu_{\eta}$. Denoting the value of the auxiliary linear program by $V_{a u x}^{w}\left(x_{n e w}, 1\right)$, again the next theorem follows from the above discussion.

Theorem 2. $V_{l p}^{w}\left(x_{n e w}, 1\right) \leq V_{a u x}^{w}\left(x_{n e w}, 1\right)$.

We now turn to the analysis of (11) which is a special case of a general linear programming problem (A.1) in Appendix A. A remark is necessary, however, in order that Proposition 1 can be applied. One assumption on the model (A.1) is that the integrands in the constraints be positive. The only integrand in (11) for which this is questionable is $\psi_{1}(x)-\left\langle\psi_{2}, \pi\right\rangle$. Recall, the solutions $\psi_{1}$ and $\psi_{2}$ are unique up to a multiplicative constant so one may multiply $\psi_{1}$ by a sufficiently large constant in order to achieve this positivity; we assume the solution $\psi_{1}$ satisfies $\left.\psi_{1}\left(x_{\text {min }}\right)\right\rangle\left\langle\psi_{2}, \pi\right\rangle$. Note this condition is satisfied when $\psi_{1}\left(x_{\min }\right)>\psi_{2}\left(x_{\max }\right)$.

Theorem 3. Assume $g_{1}$ and $g_{2}$ satisfy Condition 1. Let $F=\left\{(u, v): u \geq x_{n e w}, v \geq x_{\max }\right\}$. Then the value of the auxiliary linear program has the following bound:

$$
V_{a u x}^{w}\left(x_{n e w}, 1\right) \leq \psi_{1}\left(x_{n e w}\right) \cdot \sup _{u, v \in F} \frac{g_{1}(u) \psi_{2}(v)+\left\langle\psi_{2}, \pi\right\rangle g_{2}(v)}{\psi_{1}(u) \psi_{2}(v)} .
$$

Moreover, optimizers $\left(u_{w}^{*}, v_{w}^{*}\right)$ of the right-hand side of (12) exist in $F$ and the hitting times

$$
\theta^{*}=\inf \left\{t>0: X(t-)=u_{w}^{*}\right\} \quad \text { and } \quad \eta^{*}=\inf \left\{t \geq \theta^{*}: X(t-)=v_{w}^{*}\right\}
$$

are optimal thinning and harvesting times, respectively, for the restricted Wicksell single-cycle problem. The optimal value for the restricted problem is

$$
V_{r}^{w}\left(x_{n e w}, 1\right)=\frac{g_{1}\left(u_{w}^{*}\right) \psi_{2}\left(v_{w}^{*}\right)+\left\langle\psi_{2}, \pi\right\rangle g_{2}\left(v_{w}^{*}\right)}{\psi_{1}\left(u_{w}^{*}\right) \psi_{2}\left(v_{w}^{*}\right)} \cdot \psi_{1}\left(x_{n e w}\right)
$$

Proof. Begin by normalizing the first constraint of (11) by dividing both sides by $\psi_{1}\left(x_{n e w}\right)$. The resulting formulation then matches the general linear program (A.1) so Proposition 1 can be applied, establishing the bound (12). Now rewrite the bounding ratio in (12) as

$$
\frac{\psi_{1}\left(x_{n e w}\right)}{\psi_{1}(u)} \cdot\left[g_{1}(u)+\frac{\left\langle\psi_{2}, \pi\right\rangle}{\psi_{2}(v)} \cdot g_{2}(v)\right]
$$

from which one can see that optimizing over $v$ only involves optimizing the ratio $\frac{g_{2}(v)}{\psi_{2}(v)}$. By Condition $1(\mathrm{c})$, there exists some $\bar{x}$ for which $g_{2}(\bar{x})>0$. The function $\psi_{2}$ is strictly positive on $(0, \infty)$ so the ratio $\frac{g_{2}(\bar{x})}{\psi_{2}(\bar{x})}>0$. By continuity of $g_{2}$ and $\psi_{2}$ and Condition $1(\mathrm{~d})$, the maximum value is achieved at some location $v_{w}^{*}<\infty$.

Now turning to the optimization over $u$, we seek to maximize the ratio $\frac{g_{1}(u)+\frac{\left\langle\psi_{2}, \pi\right\rangle g_{2}\left(v_{w}^{*}\right)}{\psi_{2}\left(v_{w}^{*}\right)}}{\psi_{1}(u)}$. Condition $1(\mathrm{c})$ implies this ratio will be strictly positive for some sufficiently large value of $u$. Since $\lim _{u \rightarrow \infty} \psi_{1}(u)=\infty$, the increase of $g_{1}$ in the numerator by the constant $\frac{\left\langle\psi_{2}, \pi\right\rangle g_{2}\left(v_{w}^{*}\right)}{\psi_{2}\left(v_{w}^{*}\right)}$ does not affect the limiting value as $u \rightarrow \infty$ and hence Condition $1(\mathrm{~d})$ establishes the existence of a maximizer $u_{w}^{*}<\infty$.

Finally, using a pair $\left(u_{w}^{*}, v_{w}^{*}\right)$ of maximizers of the ratio in (12), define the decision times $\theta^{*}$ and $\eta^{*}$ as in (13). The measure $\nu_{\theta^{*}}$ will concentrate all of its mass on $\left\{u_{w}^{*}\right\}$ and similarly $\nu_{\eta^{*}}$ is a point mass on $\left\{v_{w}^{*}\right\}$. The two constraints of the auxiliary linear program (11) form the system

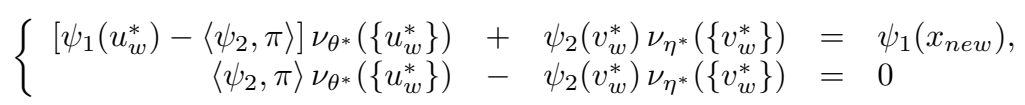

from which one readily determines that $\nu_{\theta^{*}}\left(\left\{u_{w}^{*}\right\}\right)=\frac{\psi_{1}\left(x_{n e w}\right)}{\psi_{1}\left(u_{w}^{*}\right)}$ and $\nu_{\eta^{*}}\left(\left\{v_{w}^{*}\right\}\right)=\frac{\psi_{1}\left(x_{n e w}\right)}{\psi_{1}\left(u_{w}^{*}\right)} \cdot \frac{\left\langle\psi_{2}, \pi\right\rangle}{\psi_{2}\left(v_{w}^{*}\right)}$. Hence this choice of thinning and harvesting times achieves the upper bound (14). 
Remark 1. Let $\theta_{u}=\inf \{t>0:(X(t-), Y(t-))=(u, 1)\}$ denote the first hitting time of $u$ by $X$ under dynamics given by $Y(t)=1$ when $X(0)=x_{n e w}$. It is well-known that

$$
E\left[e^{-\alpha \theta_{u}}\right]=\frac{\psi_{1}\left(x_{n e w}\right)}{\psi_{1}(u)}
$$

(see Borodin and Salminen, 2002, II.1.10, p. 18). Now let $\eta_{v}=\inf \{t>0:(X(t), Y(t))=(v, 2)\}$ be the first hitting time by $X$ of $v$ under dynamics given by $Y(t)=2$. Conditioning on $X\left(\theta_{u}\right)=x$, we have $E\left[e^{-\alpha\left(\eta_{v}-\theta_{u}\right)} \mid X\left(\theta_{u}\right)=x\right]=\psi_{2}(x) / \psi_{2}(v)$ and since $X\left(\theta_{u}\right)$ has distribution $\pi$ on $\left[x_{\text {min }}, x_{\text {max }}\right]$, it follows that $E\left[e^{-\alpha\left(\eta_{v}-\theta_{u}\right)}\right]=\left\langle\psi_{2}, \pi\right\rangle / \psi_{2}(v)$ and hence that $E\left[e^{-\alpha \eta_{v}}\right]=\psi_{1}\left(x_{n e w}\right) / \psi_{1}(u) \cdot\left\langle\psi_{2}, \pi\right\rangle / \psi_{2}(v)$. The ratio in (12) can be expressed as

$$
\begin{aligned}
\frac{\psi_{1}\left(x_{n e w}\right)}{\psi_{1}(u)} \cdot g_{1}(u)+\frac{\psi_{1}\left(x_{n e w}\right)}{\psi_{1}(u)} \cdot \frac{\left\langle\psi_{2}, \pi\right\rangle}{\psi_{2}(v)} \cdot g_{2}(v) \\
\quad=E\left[e^{-\alpha \theta_{u}} g_{1}\left(X\left(\theta_{u}-\right)\right) I_{\left\{\theta_{u}<\infty\right\}}+e^{-\alpha \eta_{v}} g_{2}\left(X\left(\eta_{v}\right)\right) I_{\left\{\eta_{v}<\infty\right\}}\right]
\end{aligned}
$$

Thus the nonlinear optimization problem (12) maximizes (2) over the hitting times of the paired process $(X, Y)$ at thinning and harvesting levels $(u, 1)$ and $(v, 2)$, respectively.

Remark 2. Let $d_{1}(u)=E\left[e^{-\alpha \theta_{u}}\right]=\psi_{1}\left(x_{n e w}\right) / \psi_{1}(u)$ denote the expected discount factor arising from the rule to thin the forest when $(X, Y)$ reaches level $(u, 1)$ and let $d_{2}(v)=E\left[e^{-\alpha\left(\eta_{v}-\theta_{u}\right)}\right]=\left\langle\psi_{2}, \pi\right\rangle / \psi_{2}(v)$ be the expected discount factor associated with the rule to harvest when $(X, Y)$ hits $(v, 2)$, starting at time $\theta_{u}$ in location $X\left(\theta_{u}\right)$. The bound (12) then takes the form $d_{1}(u)\left[g_{1}(u)+d_{2}(v) g_{2}(v)\right]$. The first order optimality conditions are therefore

$$
\left\{\begin{aligned}
d_{1}^{\prime}(u)\left[g_{1}(u)+d_{2}(v) g_{2}(v)\right]+d_{1}(u) g_{1}^{\prime}(u) & =0 \\
d_{2}^{\prime}(v) g_{2}(v)+d_{2}(v) g_{2}^{\prime}(v) & =0
\end{aligned}\right.
$$

which can be rewritten as

$$
\left\{\begin{aligned}
\frac{u \frac{\partial}{\partial u}\left[g_{1}(u)+d_{2}(v) g_{2}(v)\right]}{\left[g_{1}(u)+d_{2}(v) g_{2}(v)\right]} & =-\frac{u d_{1}^{\prime}(u)}{d_{1}(u)} \\
\frac{v g_{2}^{\prime}(v)}{g_{2}(v)} & =-\frac{v d_{2}^{\prime}(v)}{d_{2}(v)} .
\end{aligned}\right.
$$

The second equation shows that the optimal harvesting level $v_{w}^{*}$ occurs where the elasticity of the harvest payoff function $g_{2}$ equals the negative of the elasticity of the harvesting discount factor $d_{2}$. The optimal thinning level $u_{w}^{*}$, however, occurs where the partial elasticity of the combined payoffs for thinning $g_{1}$ and discounted harvesting $d_{2}(v) g_{2}(v)$ equals the negative of the elasticity of the thinning discount factor $d_{1}$.

\subsection{Optimality for the Unrestricted Problem}

The results of Section 2.2 determine an optimal pair of thinning and harvesting times $\left(\theta^{*}, \eta^{*}\right) \in \mathcal{A}_{1}$ for the restricted problem. This subsection demonstrates that this pair of decision times is also optimal for the unrestricted problem.

Theorem 4. Assume $g_{1}$ and $g_{2}$ satisfy Condition 1. Then $V^{w}\left(x_{n e w}, 1\right)=V_{r}^{w}\left(x_{n e w}, 1\right)$ and hence the thinning and harvest times $\left(\theta^{*}, \eta^{*}\right)$ of Theorem 3 is an optimal pair for the unrestricted stochastic forestry problem.

Proof. Choose $(\theta, \eta) \in \mathcal{A}$ arbitrarily. Select a sequence $K_{n} \nearrow \infty$ and define $\tau_{K_{n}}=\inf \left\{t>0: X(t)=K_{n}\right\}$. Since $\infty$ is a natural boundary point, it follows that $\tau_{K_{n}} \nearrow \infty$ as $n \rightarrow \infty$. Observe that $\left\{\left(\theta \wedge \tau_{K_{n}}, \eta \wedge \tau_{K_{N}}\right)\right\}$ is a sequence of decision rules within the restricted collection $\mathcal{A}_{1}$ and hence

$$
V_{r}^{w}\left(x_{n e w}, 1\right) \geq E\left[e^{-\alpha\left(\theta \wedge \tau_{K_{n}}\right)} g_{1}\left(X\left(\theta \wedge \tau_{K_{n}}\right)\right) I_{\left.\left\{\theta \wedge \tau_{K_{n}}\right)<\infty\right\}}\right.
$$




$$
\begin{gathered}
\left.\quad+e^{-\alpha\left(\eta \wedge \tau_{K_{n}}\right)} g_{2}\left(X\left(\eta \wedge \tau_{K_{n}}\right)\right) I_{\left.\left\{\eta \wedge \tau_{K_{n}}\right)<\infty\right\}}\right] \\
=E\left[e^{-\alpha\left(\theta \wedge \tau_{K_{n}}\right)} g_{1}\left(X\left(\theta \wedge \tau_{K_{n}}\right)\right) I_{\left.\left\{\theta \wedge \tau_{K_{n}}\right)<\infty\right\}} I_{\{\theta<\infty\}}\right] \\
+E\left[e^{-\alpha\left(\theta \wedge \tau_{K_{n}}\right)} g_{1}\left(X\left(\theta \wedge \tau_{K_{n}}\right)\right) I_{\left.\left\{\theta \wedge \tau_{K_{n}}\right)<\infty\right\}} I_{\{\theta=\infty\}}\right] \\
+E\left[e^{-\alpha\left(\eta \wedge \tau_{K_{n}}\right)} g_{2}\left(X\left(\eta \wedge \tau_{K_{n}}\right)\right) I_{\left\{\eta \wedge \tau_{K_{n}}<\infty\right\}} I_{\{\eta<\infty\}}\right] \\
+E\left[e^{-\alpha\left(\eta \wedge \tau_{K_{n}}\right)} g_{2}\left(X\left(\eta \wedge \tau_{K_{n}}\right)\right) I_{\left\{\eta \wedge \tau_{K_{n}}<\infty\right\}} I_{\{\eta=\infty\}}\right] .
\end{gathered}
$$

Consider the first expectation on the right-hand side of (17). Observe that on the set where $\theta$ is finite, i. e. $\{\theta<\infty\}, \theta(\omega) \wedge \tau_{K_{n}}(\omega)=\theta(\omega)$ for $n$ sufficiently large. Thus

$$
\lim _{n \rightarrow \infty} e^{-\alpha\left(\theta \wedge \tau_{K_{n}}\right)} g_{1}\left(X\left(\theta \wedge \tau_{K_{n}}\right)\right) I_{\left\{\theta \wedge \tau_{K_{n}}<\infty\right\}} I_{\{\theta<\infty\}}=e^{-\alpha \theta} g_{1}(X(\theta)) I_{\{\theta<\infty\}}
$$

and hence Fatou's lemma implies that

$$
E\left[e^{-\alpha \theta} g_{1}(X(\theta)) I_{\{\theta<\infty\}}\right] \leq \lim _{n \rightarrow \infty} E\left[e^{-\alpha\left(\theta \wedge \tau_{K_{n}}\right)} g_{1}\left(X\left(\theta \wedge \tau_{K_{n}}\right)\right) I_{\left\{\theta \wedge \tau_{K_{n}}<\infty\right\}} I_{\{\theta<\infty\}}\right] .
$$

Analyzing the second term, notice that on the set $\{\theta=\infty\}, \theta \wedge \tau_{K_{n}}=\tau_{K_{n}}$ so

$$
\begin{aligned}
E\left[e^{-\alpha\left(\theta \wedge \tau_{K_{n}}\right)} g_{1}\left(X\left(\theta \wedge \tau_{K_{n}}\right)\right) I_{\left\{\theta \wedge \tau_{K_{n}}\right\}} I_{\{\theta=\infty\}}\right] & =E\left[e^{-\alpha \tau_{K_{n}}} g_{1}\left(X\left(\tau_{K_{n}}\right)\right) I_{\left\{\tau_{K_{n}}<\infty\right\}} I_{\{\theta=\infty\}}\right] \\
& \leq g_{1}\left(K_{n}\right) E\left[e^{-\alpha \tau_{K_{n}}} I_{\left\{\tau_{K_{n}}<\infty\right\}}\right] \\
& =g_{1}\left(K_{n}\right) \cdot \frac{\psi_{1}\left(x_{n e w}\right)}{\psi_{1}\left(K_{n}\right)}
\end{aligned}
$$

and the right-hand side converges to 0 by Condition $1(\mathrm{~d})$. The same analyses applies to the third and fourth expectations in (17) with the result that

$$
V_{r}^{w}\left(x_{n e w}, 1\right) \geq E\left[e^{-\alpha \theta} g_{1}(X(\theta)) I_{\{\theta<\infty\}}+e^{-\alpha \eta} g_{2}(X(\eta)) I_{\{\eta<\infty\}}\right] .
$$

Taking the supremum over $(\theta, \eta) \in \mathcal{A}$ implies $V_{r}^{w}\left(x_{n e w}, 1\right) \geq V^{w}\left(x_{n e w}, 1\right)$. The reverse inequality follows immediately from the fact that $\mathcal{A}_{1} \subset \mathcal{A}$.

\subsection{Example}

For simplicity, we illustrate the single cycle thinning-and-harvesting problem by looking at the case in which $X$ is a mean reverting process for both dense and thinned forests. The key feature of this model are particular mean levels for dense and thinned forests to which the process is attracted. In our formulation, the mean levels are $1 / \gamma_{y}$, with $\gamma_{y}>0$. When the process is smaller than $1 / \gamma_{y}$, the drift will be positive, but when the process exceeds this level, the drift will be negative. These levels therefore act as natural targets for the sizes of the dense and thinned forest or for the size of an individual tree in a dense or thinned forest, depending on what the process $X$ models for each type of forest. Specifically, the process $X$ satisfies (1), where for $y=1,2, \mu(x, y)=\bar{\mu}\left(1-\gamma_{y} x\right)$ and $\sigma(x, y)=\bar{\sigma} \sqrt{x}$, with $\bar{\mu}$ and $\bar{\sigma}$ being fixed positive constants. This model is an example of a process on the state space $(0, \infty)$ for which the left end-point 0 is an entrancenot-exit boundary point. For simplicity, we take the distribution $\pi$ of the size of the thinned forest stand $X(\theta)$ to be a unit point mass at $x_{\text {thin }}$ which means $X(\theta)=x_{\text {thin }}$ following the decision to thin.

The differential operators for the mean-reverting model are $A^{(y)} f(x)=\bar{\mu}\left(1-\gamma_{y} x\right) f^{\prime}(x)+\left(\bar{\sigma}^{2} / 2\right) x f^{\prime \prime}(x)$, $y=1,2$. The increasing solutions of the eigenfunction equation (5) are given by

$$
\psi_{y}(x)=K_{M}\left(\frac{\alpha}{\gamma_{y} \bar{\mu}}, \frac{2 \bar{\mu}}{\bar{\sigma}^{2}}, \frac{2 \gamma_{y} \bar{\mu}}{\bar{\sigma}^{2}} x\right),
$$


in which $K_{M}(a, b, z)$ denotes the Kummer $M$-function

$$
K_{M}(a, b, z)=1+\frac{a z}{b}+\frac{(a)_{2}}{(b)_{2}} \frac{z^{2}}{2 !}+\cdots+\frac{(a)_{n}}{(b)_{n}} \frac{z^{n}}{n !}+\cdots
$$

where for $c=a, b,(c)_{n}:=c(c+1)(c+2) \cdot \ldots \cdot(c+n-1),(c)_{0}:=1$ (see Abramowitz and Stegun (1965) for details). For instance, $K_{M}(a, b, z)$ is a solution of the ordinary differential equation

$$
z f^{\prime \prime}(z)+(b-z) f^{\prime}(z)-a f(z)=0
$$

an alternative notation for the Kummer $M$-function is $K_{M}(a, b, z)={ }_{1} F_{1}(a, b ; z)$ in Abramowitz and Stegun (1965).

For illustrative purposes we choose the thinning and harvesting reward functions $g_{1}$ and $g_{2}$ as follows:

$$
g_{y}(x)=x \cdot \delta_{y} \cdot \frac{1+\tanh \left(\varrho_{y}\left(x-\mathfrak{z}_{y}\right)\right)}{2}-c_{y}, \quad y=1,2,
$$

where for $y=1$ and $2, \delta_{y}, \varrho_{y}, \mathfrak{z}_{y}$ and $c_{y}$ are nonnegative constants. Nice interpretations can be given to the parameters: $\delta_{y}$ represents the long-term growth rate of timber values (for large values of $x$ ) when $\varrho>0$; the quantity $c_{y}$ may include a common shift in value that applies to all timber sizes (e.g., property tax or transportation costs per tree Davies (1996), Table 1); $\varrho_{y}$ is a scaling factor and $\mathfrak{z} y$ is close to the inflection point where profits increase the most. This form for the harvesting payoff function $g_{2}$ gives a reasonable family of functions that provide good approximations to the "Tree Value Conversion Standards" (see Mendel, DeBald, and Dale (1976)) which estimate the harvesting payoffs based on the size (diameter at breast height - dbh) and grade of the tree. An example of the fit for sugar maples is provided in Appendix B; appropriate choices of parameters also provide good fits of $g_{2}$ for the TVCS of other tree varieties as well. (The tree value conversion standards were revisited in the publication DeBald and Dale (1991); we base our illustration on the original paper by Mendel, DeBald, and Dale (1976).) The form (19) of payoff function can be used for $g_{1}$ to obtain good approximations of the "Fuel Value" (see Morrow (1981)). Notice that this class of functions includes affine functions when $\varrho=0$.

The paper Morrow (1981) lists the TVCS values corresponding to hardwood trees having dbh in the range from 10 inches to 28 inches and comments that trees with dbh in the range of 4 to 10 inches are worth nothing except for fuelwood, but are in the most need of thinning. He further adds that trees with dbh in the range of 10-14 inches have marginal value for timber and the rate of value increase is high, especially for thinned trees. The situation is reversed when the trees have dbh in the range 24 to 28 inches with high values but low growth rates. The function class (19) for $g_{2}$ captures this type of value change while appropriate choices of $\gamma_{y}$ in the mean-reverting stochastic growth model will provide the observed type of growth. Morrow also indicates that trees with dbh of 20 inches "do not just occur; they are the result of thinning young stands, good sites, or a combination of both."

Table 1 illustrates the optimal thinning and harvesting levels $u_{w}^{*}$ and $v_{w}^{*}$, respectively, for a particular choice of parameters along with the optimal expected discounted payoff $V^{w}\left(x_{n e w}, 1\right)$ for thinning and harvesting. The particular choice of $\varrho_{1}=0$ and $\mathfrak{z}_{1}=0$ indicates that a good fit to the fuelwood values is given by an affine function. First observe that the value of $g_{1}\left(u_{w}^{*}\right)$ is slightly negative indicating that it is optimal to spend money to thin the forest to receive the benefit of larger and more valuable trees when one harvests. Thus thinning is important in order to develop more valuable trees rather than as a source of income. Notice also the effect of increasing the thinned size of the forest is to thin earlier but this has no impact on the decision to harvest. Intuitively, when $x_{\text {thin }}$ is small, the decision to thin would remove larger trees so as to allow smaller, good quality trees to grow to full size and be harvested. When $x_{\text {thin }}$ is large, however, the results indicate that the smaller competing trees would be thinned leaving larger trees for later harvest.

These values in Table 1 should be compared with those when thinning is not applied and the process $X$ only evolves according to the mean-reverting regime with parameters $\mu=1, \sigma^{2}=0.03$ and $1 / \gamma_{1}=100$, and the reward function is given by $g_{2}$. The choice of $g_{2}$ for this comparison provides an optimistic value since the trees in a dense forest will not grow to the same size or grade as for a thinned forest, but there will be some trees of sufficient size so that the use of the fuelwood payoff function $g_{1}$ would not be appropriate. The 


$\begin{array}{lccc}x_{\text {thin }} & u_{w}^{*} & v_{w}^{*} & V^{w}\left(x_{\text {new }}, 1\right) \\ 10 . & 29.3 & 61.7 & 3.257 \\ 12.5 & 28.0 & 61.7 & 3.493 \\ 15 . & 26.5 & 61.7 & 3.770 \\ 17.5 & 24.9 & 61.7 & 4.097 \\ 20 . & 23.1 & 61.7 & 4.487 \\ 22.5 & 21.0 & 61.7 & 4.957 \\ 25 . & 18.8 & 61.7 & 5.526\end{array}$

Table 1: Optimal thinning levels $u_{w}^{*}$ and harvesting levels $v_{w}^{*}$ (in $\mathrm{cm}$ ) for the Wicksell-Model as well as values of $V^{w}\left(x_{n e w}, 1\right)$ for various $x_{\text {thin }}$ values (in cm) using two mean reverting processes; $\mu_{1}=\mu_{2}=1, \sigma_{1}^{2}=\sigma_{2}^{2}=0.03 ; 1 / \gamma_{1}=100,1 / \gamma_{2}=120$, $\alpha=0.03, x_{n e w}=0.5, \delta_{1}=0.7345, \varrho_{1}=0, \mathfrak{z}_{1}=0, c_{1}=9.1748, \delta_{2}=1.8254, \varrho_{2}=0.04502, \mathfrak{z}_{2}=56.6523, c_{2}=4.3862$.

optimal harvesting level of the corresponding Wicksell problem equals 58.8 and the optimal value is 4.47 . Moreover, the expected harvesting time is $\sim 87$ years. For the case with thinning, when $x_{\text {thin }}=20 \mathrm{~cm}$ or $x_{\text {thin }}=22.5 \mathrm{~cm}$ in Table 1 (and the associated selection of parameters and payoff functions), for example, the two phases - before thinning and after thinning - have an average length of 22-25 years and 61-64 years respectively. Hence, thinning might slightly increase the average optimal cash flow while the average time up to harvesting is about the same. (The integral formula for the mean hitting time $\tau_{b}$ of a level $b>x_{0}$ of a mean-reverting process is $E\left[\tau_{b}\right]=\int_{x_{0}}^{b} \int_{0}^{v} e^{-\int_{z}^{v} \frac{2 \mu(u, y)}{\sigma^{2}(u, y)} d u} \cdot \frac{2}{\sigma^{2}(z, y)} d z d v$, with $\mu(x, y)$ and $\sigma(x, y)$ specified at the beginning of this example (Karlin and Taylor, 1981, pp. 192-197); this formula has been used to determine these mean thinning and harvest times.)

The optimal values $u_{w}^{*}, v_{w}^{*}$ and thinning level $x_{\text {thin }}$ serve as recommendations to the forester who would be given the task of determining how to implement thinning and harvesting. Additional comments about the model and implementation are given in Section 4 of concluding remarks.

\section{Faustmann Infinite-Cycle Rotation Problem}

In contrast with the Wicksell problem whose goal is to maximize the expected discounted reward for thinning and harvesting a forest one time, the Faustmann problem allows the forest to be replanted after each harvest and therefore rewards are earned over an infinite number of thinning and harvesting cycles.

\subsection{Reduction to a Wicksell Single-Cycle Problem}

This section analyzes the Faustmann problem by using the strong Markov property to relate it to a Wicksell problem for an adjusted payoff function.

We assume the dynamics of the forest growth process $X$ follow (1), that thinning occurs at times $\left\{\theta_{k}\right\}$ with the thinned state $X\left(\theta_{k}\right)$ having distriubtion $\pi$ and that harvesting and replanting happens at times $\eta_{k}$ at which point the process $X$ reinitializes at $x_{n e w}$. The Faustmann objective is to maximize (3) over all $\left\{\left(\theta_{k}, \eta_{k}\right)\right\} \in \mathcal{A}$. Note, we assume $\theta_{k} \leq \eta_{k} \leq \theta_{k+1}$ for every $k$. Recall $V^{f}\left(x_{n e w}, 1\right)$ denotes the optimal value for the Faustmann problem.

To facilitate understanding of the argument for this section, we use a subscript on the expectation operator, e.g. $E_{x_{n e w}}[\cdot]$, to indicate the initial position of the process $X$. This notation is important when using the strong Markov property.

Theorem 5. Assume $g_{1}$ and $g_{2}$ satisfy Condition 1. Then the optimal value for the Faustmann infinite thinning-and-harvesting cycle problem is characterized by the nonlinear optimization problem

$$
V^{f}\left(x_{n e w}, 1\right)=\sup _{u, v \in F} \frac{g_{1}(u) \psi_{2}(v)+\left\langle\psi_{2}, \pi\right\rangle g_{2}(v)}{\psi_{1}(u) \psi_{2}(v)-\psi_{1}\left(x_{n e w}\right)\left\langle\psi_{2}, \pi\right\rangle} \cdot \psi_{1}\left(x_{\text {new }}\right) .
$$

Moreover, an optimal pair $\left(u_{f}^{*}, v_{f}^{*}\right)$ exists and the thinning and harvest times are given by the successive hitting times of $X$ to the levels $u_{f}^{*}$ and $v_{f}^{*}$, namely, setting $\eta_{0}^{*}=0$, define, for $k=1,2,3, \ldots$,

$$
\theta_{k}^{*}=\inf \left\{t>\eta_{k-1}^{*}: X(t-)=u_{f}^{*}\right\} \quad \text { and } \quad \eta_{k}^{*}=\inf \left\{t>\theta_{k}^{*}: X(t-)=v_{f}^{*}\right\} .
$$


The optimal value is therefore

$$
V^{f}\left(x_{n e w}, 1\right)=\frac{g_{1}\left(u_{f}^{*}\right) \psi_{2}\left(v_{f}^{*}\right)+\left\langle\psi_{2}, \pi\right\rangle g_{2}\left(v_{f}^{*}\right)}{\psi_{1}\left(u_{f}^{*}\right) \psi_{2}\left(v_{f}^{*}\right)-\psi_{1}\left(x_{n e w}\right)\left\langle\psi_{2}, \pi\right\rangle} \cdot \psi_{1}\left(x_{n e w}\right) .
$$

Proof. In preparation for the analysis, notice that for $k \geq 2, \theta_{k} \geq \eta_{1}$; define $\tilde{\theta}_{k-1}=\theta_{k}-\eta_{1}$. Also recall that on the set $\left\{\eta_{1}<\infty\right\}, X\left(\eta_{1}\right)=x_{n e w}$ and observe that the summands of (3) are 0 for $k \geq 2$ on the set $\left\{\eta_{1}=\infty\right\}$. Then using the strong Markov property in the second last equality below, we have

$$
\begin{aligned}
E_{x_{\text {new }}} & {\left[\sum_{k=1}^{\infty} e^{-\alpha \theta_{k}} g_{1}\left(X\left(\theta_{k}-\right)\right) I_{\left\{\theta_{k}<\infty\right\}}\right] } \\
= & E_{x_{\text {new }}}\left[e^{-\alpha \theta_{1}} g_{1}\left(X\left(\theta_{1}-\right)\right) I_{\left\{\theta_{1}<\infty\right\}}\right]+E_{x_{n e w}}\left[\sum_{k=2}^{\infty} e^{-\alpha \theta_{k}} g_{1}\left(X\left(\theta_{k}-\right)\right) I_{\left\{\theta_{k}<\infty\right\}}\right] \\
= & E_{x_{n e w}}\left[e^{-\alpha \theta_{1}} g_{1}\left(X\left(\theta_{1}-\right)\right) I_{\left\{\theta_{1}<\infty\right\}}\right] \\
& +E_{x_{n e w}}\left[E_{x_{n e w}}\left[e^{-\alpha \eta_{1}} I_{\left\{\eta_{1}<\infty\right\}} \sum_{k=2}^{\infty} e^{-\alpha\left(\theta_{k}-\eta_{1}\right)} g_{1}\left(X\left(\eta_{1}+\left(\theta_{k}-\eta_{1}\right)-\right)\right) I_{\left\{\eta_{1}+\left(\theta_{k}-\eta_{1}\right)<\infty\right\}} \mid \mathcal{F}_{\eta_{1}}\right]\right] \\
= & E_{x_{n e w}}\left[e^{-\alpha \theta_{1}} g_{1}\left(X\left(\theta_{1}-\right)\right) I_{\left\{\theta_{1}<\infty\right\}}\right] \\
& +E_{x_{n e w}}\left[e^{-\alpha \eta_{1}} I_{\left\{\eta_{1}<\infty\right\}} E_{x_{n e w}}\left[\sum_{k=1}^{\infty} e^{-\alpha \tilde{\theta}_{k}} g_{1}\left(X\left(\eta_{1}+\tilde{\theta}_{k}-\right)\right) I_{\left\{\eta_{1}+\tilde{\theta}_{k}<\infty\right\}} \mid \mathcal{F}_{\eta_{1}}\right]\right] \\
= & E_{x_{n e w}}\left[e^{-\alpha \theta_{1}} g_{1}\left(X\left(\theta_{1}-\right)\right) I_{\left\{\theta_{1}<\infty\right\}}\right] \\
& +E_{x_{n e w}}\left[e^{-\alpha \eta_{1}} I_{\left\{\eta_{1}<\infty\right\}} E_{X\left(\eta_{1}\right)}\left[\sum_{k=1}^{\infty} e^{-\alpha \tilde{\theta}_{k}} g_{1}\left(X\left(\tilde{\theta}_{k}-\right)\right) I_{\left\{\tilde{\theta}_{k}<\infty\right\}}\right]\right] \\
= & E_{x_{n e w}}\left[e^{-\alpha \theta_{1}} g_{1}\left(X\left(\theta_{1}-\right)\right) I_{\left\{\theta_{1}<\infty\right\}}\right] \\
& +E_{x_{n e w}}\left[e^{-\alpha \eta_{1}} I_{\left\{\eta_{1}<\infty\right\}} E_{x_{n e w}}\left[\sum_{k=1}^{\infty} e^{-\alpha \tilde{\theta}_{k}} g_{1}\left(X\left(\tilde{\theta}_{k}-\right)\right) I_{\left\{\tilde{\theta}_{k}<\infty\right\}}\right]\right] .
\end{aligned}
$$

Define $\tilde{\eta}_{k-1}=\eta_{k}-\eta_{1}$, for $k \geq 2$. The same analysis can be applied to the rewards obtained from harvesting and thus

$$
\begin{aligned}
E_{x_{\text {new }}} & {\left[\sum_{k=1}^{\infty}\left(e^{-\alpha \theta_{k}} g_{1}\left(X\left(\theta_{k}-\right)\right) I_{\left\{\theta_{k}<\infty\right\}}+e^{-\alpha \eta_{k}} g_{2}\left(X\left(\eta_{k}-\right)\right) I_{\left\{\eta_{k}<\infty\right\}}\right)\right] } \\
= & E_{x_{n e w}}\left[e^{-\alpha \theta_{1}} g_{1}\left(X\left(\theta_{1}-\right)\right) I_{\left\{\theta_{1}<\infty\right\}}+e^{-\alpha \eta_{1}} g_{2}\left(X\left(\eta_{1}-\right)\right) I_{\left\{\eta_{1}<\infty\right\}}\right. \\
& \left.+e^{-\alpha \eta_{1}} I_{\left\{\eta_{1}<\infty\right\}} E_{x_{n e w}}\left[\sum_{k=1}^{\infty}\left(e^{-\alpha \tilde{\theta}_{k}} g_{1}\left(X\left(\tilde{\theta}_{k}-\right)\right) I_{\left\{\tilde{\theta}_{k}<\infty\right\}}+e^{-\alpha \tilde{\eta}_{k}} g_{2}\left(X\left(\tilde{\eta}_{k}-\right)\right) I_{\left\{\tilde{\eta}_{k}<\infty\right\}}\right)\right]\right] .
\end{aligned}
$$

Denote the infinite sequences by $(\tilde{\theta}, \tilde{\eta})=\left\{\left(\tilde{\theta}_{k}, \tilde{\eta}_{k}\right)\right\}$ and notice $(\tilde{\theta}, \tilde{\eta}) \in \mathcal{A}$. Observe

$$
\begin{aligned}
& V^{f}\left(x_{n e w}, 1\right) \\
& \quad=\sup _{(\tilde{\theta}, \tilde{\eta}) \in \mathcal{A}} E_{x_{n e w}}\left[\sum_{k=1}^{\infty}\left(e^{-\alpha \tilde{\theta}_{k}} g_{1}\left(X\left(\tilde{\theta}_{k}-\right)\right) I_{\left\{\tilde{\theta}_{k}<\infty\right\}}+e^{-\alpha \tilde{\eta}_{k}} g_{2}\left(X\left(\tilde{\eta}_{k}-\right)\right) I_{\left\{\tilde{\eta}_{k}<\infty\right\}}\right)\right] .
\end{aligned}
$$

Taking the supremum of the right-hand side of $(23)$ over $(\tilde{\theta}, \tilde{\eta}) \in \mathcal{A}$ implies

$$
E_{x_{n e w}}\left[\sum_{k=1}^{\infty}\left(e^{-\alpha \theta_{k}} g_{1}\left(X\left(\theta_{k}-\right)\right) I_{\left\{\theta_{k}<\infty\right\}}+e^{-\alpha \eta_{k}} g_{2}\left(X\left(\eta_{k}-\right)\right) I_{\left\{\eta_{k}<\infty\right\}}\right)\right]
$$




$$
\begin{aligned}
& \leq E_{x_{\text {new }}}\left[e^{-\alpha \theta_{1}} g_{1}\left(X\left(\theta_{1}-\right)\right) I_{\left\{\theta_{1}<\infty\right\}}+e^{-\alpha \eta_{1}} g_{2}\left(X\left(\eta_{1}-\right)\right) I_{\left\{\eta_{1}<\infty\right\}}+e^{-\alpha \eta_{1}} I_{\left\{\eta_{1}<\infty\right\}} V^{f}\left(x_{n e w}, 1\right)\right] \\
& =E_{x_{\text {new }}}\left[e^{-\alpha \theta_{1}} g_{1}\left(X\left(\theta_{1}-\right)\right) I_{\left\{\theta_{1}<\infty\right\}}+e^{-\alpha \eta_{1}}\left[g_{2}\left(X\left(\eta_{1}-\right)\right)+V^{f}\left(x_{n e w}, 1\right)\right] I_{\left\{\eta_{1}<\infty\right\}}\right] \\
& =E_{x_{\text {new }}}\left[e^{-\alpha \theta_{1}} g_{1}\left(X\left(\theta_{1}-\right)\right) I_{\left\{\theta_{1}<\infty\right\}}+e^{-\alpha \eta_{1}} \tilde{g}_{2}\left(X\left(\eta_{1}-\right)\right) I_{\left\{\eta_{1}<\infty\right\}}\right]
\end{aligned}
$$

where $\tilde{g}_{2}(x)=g_{2}(x)+V^{f}\left(x_{n e w}, 1\right)$. Thus the Faustmann infinite-cycle rotation problem is bounded above by a Wicksell single-cycle problem using the thinning payoff function $g_{1}$ and a shifted harvesting payoff function $\tilde{g}_{2}$. In a similar manner, begin by taking the supremum of the left-hand side of $(23)$ over $(\theta, \eta) \in \mathcal{A}$, then the supremum of the right-hand side over $(\tilde{\theta}, \tilde{\eta}) \in \mathcal{A}$ and finally the supremum of the right-hand side over $\left(\theta_{1}, \eta_{1}\right)$ in the Wicksell admissible set to obtain the opposite inequality and hence the value $V^{f}\left(x_{n e w}, 1\right)$ equals the value $\tilde{V}^{w}\left(x_{n e w}, 1\right)$ of this Wicksell problem. Theorem 3 establishes existence of optimizers $u_{f}^{*}$ and $v_{f}^{*}$ for which the value of this Wicksell problem (and hence the Faustmann problem) is

$$
V^{f}\left(x_{n e w}, 1\right)=\tilde{V}^{w}\left(x_{n e w}, 1\right)=\frac{g_{1}\left(u_{f}^{*}\right) \psi_{2}\left(v_{f}^{*}\right)+\left\langle\psi_{2}, \pi\right\rangle \tilde{g}_{2}\left(v_{f}^{*}\right)}{\psi_{1}\left(u_{f}^{*}\right) \psi_{2}\left(v_{f}^{*}\right)} \cdot \psi_{1}\left(x_{n e w}\right)
$$

Substituting $\tilde{g}_{2}\left(v_{f}^{*}\right)=g_{2}\left(v_{f}^{*}\right)+V^{f}\left(x_{n e w}, 1\right)$ into $(24)$ and solving for $V^{f}\left(x_{n e w}, 1\right)$ yields

$$
\begin{aligned}
V^{f}\left(x_{\text {new }}, 1\right) & =\frac{\psi_{1}\left(x_{\text {new }}\right)\left[g_{1}\left(u_{f}^{*}\right) \psi_{2}\left(v_{f}^{*}\right)+\psi_{2}\left(x_{\text {thin }}\right) g_{2}\left(v_{f}^{*}\right)\right]}{\psi_{1}\left(u_{f}^{*}\right) \psi_{2}\left(v_{f}^{*}\right)} \cdot\left[1-\frac{\psi_{1}\left(x_{\text {new }}\right)\left\langle\psi_{2}, \pi\right\rangle}{\psi_{1}\left(u_{f}^{*}\right) \psi_{2}\left(v_{f}^{*}\right)}\right]^{-1} \\
& =\frac{g_{1}\left(u_{f}^{*}\right) \psi_{2}\left(v_{f}^{*}\right)+\psi_{2}\left(x_{\text {thin }}\right) g_{2}\left(v_{f}^{*}\right)}{\psi_{1}\left(u_{f}^{*}\right) \psi_{2}\left(v_{f}^{*}\right)-\psi_{1}\left(x_{\text {new }}\right)\left\langle\psi_{2}, \pi\right\rangle} \cdot \psi_{1}\left(x_{\text {new }}\right) .
\end{aligned}
$$

Now define $(\theta, \eta) \in \mathcal{A}$ to be the successive hitting times of $u_{f}^{*}$ and $v_{f}^{*}$ defined in $(21)$. Then $X\left(\theta_{k}^{*}-\right)=u_{f}^{*}$ and $X\left(\eta_{k}^{*}-\right)=v_{f}^{*}$ and the evaluation of (3) then involves determining the value of the infinite series comprised of the Laplace transform of the successive hitting times of the levels $u_{f}^{*}$ or $v_{f}^{*}$. The evaluation of these series is given in Appendix $\mathrm{C}$ with the result that the expected discounted reward obtained using this policy achieves the bound (25).

The final point to be addressed is the demonstration that $\left(u_{f}^{*}, v_{f}^{*}\right)$ is an optimizing pair for the nonlinear optimization problem (20). To see this, the equality of $V^{f}\left(x_{n e w}, 1\right)$ and $\tilde{V}^{w}\left(x_{n e w}, 1\right)$ in $(24)$ implies that

$$
V^{f}\left(x_{n e w}, 1\right)=\sup _{u, v \in F} \frac{g_{1}(u) \psi_{2}(v)+\left\langle\psi_{2}, \pi\right\rangle \tilde{g}_{2}(v)}{\psi_{1}(u) \psi_{2}(v)} \cdot \psi_{1}\left(x_{n e w}\right)
$$

and hence for each $u, v \in F$,

$$
\begin{aligned}
V^{f}\left(x_{n e w}, 1\right) & \geq \frac{g_{1}(u) \psi_{2}(v)+\left\langle\psi_{2}, \pi\right\rangle \tilde{g}_{2}(v)}{\psi_{1}(u) \psi_{2}(v)} \cdot \psi_{1}\left(x_{\text {new }}\right) \\
& =\frac{g_{1}(u) \psi_{2}(v)+\left\langle\psi_{2}, \pi\right\rangle g_{2}(v)}{\psi_{1}(u) \psi_{2}(v)} \cdot \psi_{1}\left(x_{\text {new }}\right)+\frac{\psi_{1}\left(x_{\text {new }}\right)\left\langle\psi_{2}, \pi\right\rangle}{\psi_{1}(u) \psi_{2}(v)} \cdot V^{f}\left(x_{\text {new }}, 1\right) .
\end{aligned}
$$

Solving for $V^{f}\left(x_{n e w}, 1\right)$ yields for each $u, v \in F$,

$$
V^{f}\left(x_{n e w}, 1\right) \geq \frac{g_{1}(u) \psi_{2}(v)+\left\langle\psi_{2}, \pi\right\rangle g_{2}(v)}{\psi_{1}\left(u_{2}^{\psi}(v)-\psi_{1}\left(x_{n e w}\right)\left\langle\psi_{2}, \pi\right\rangle\right.} \cdot \psi_{1}\left(x_{n e w}\right)
$$

with equality for $\left(u_{f}^{*}, v_{f}^{*}\right)$, establishing $(20)$.

Remark 3. A similar remark to that of Remark 2 holds for the Faustmann problem. Specifically, let

$$
d_{1}(u, v)=\frac{\psi_{1}\left(x_{n e w}\right) \psi_{2}(v)}{\psi_{1}(u) \psi_{2}(v)-\psi_{1}\left(x_{n e w}\right)\left\langle\psi_{2}, \pi\right\rangle} \text { and } d_{2}(u, v)=\frac{\psi_{1}\left(x_{n e w}\right)\left\langle\psi_{2}, \pi\right\rangle}{\psi_{1}(u) \psi_{2}(v)-\psi_{1}\left(x_{n e w}\right)\left\langle\psi_{2}, \pi\right\rangle}
$$


denote the sums of the expected thinning discount factors and the expected harvesting discount factors, respectively, when thinning occurs at level $u$ and harvesting occurs at level $v$. Observe the nonlinear function $(20)$ to be maximized takes the form $d_{1}(u, v) g_{1}(u)+d_{2}(u, v) g_{2}(v)$. Also note that $d_{1}(u, v)=\frac{\psi_{2}(v)}{\left\langle\psi_{2}, \pi\right\rangle} \cdot d_{2}(u, v)$. Letting $g(u, v)=\frac{\psi_{2}(v)}{\left\langle\psi_{2}, \pi\right\rangle} \cdot g_{1}(u)+g_{2}(v)$, (20) can be written as $d_{2}(u, v) g(u, v)$. The first order optimality conditions for $\left(u_{f}^{*}, v_{f}^{*}\right)$ yield

$$
\left\{\begin{array}{l}
\frac{u_{f}^{*} \frac{\partial g}{\partial u}\left(u_{f}^{*}, v_{f}^{*}\right)}{g\left(u_{f}^{*}, v_{f}^{*}\right)}=-\frac{u_{f}^{*} \frac{\partial d_{2}}{\partial u}\left(u_{f}^{*}, v_{f}^{*}\right)}{d_{2}\left(u_{f}^{*}, v_{f}^{*}\right)} \\
\frac{v_{f}^{*} \frac{\partial g}{\partial v}\left(u_{f}^{*}, v_{f}^{*}\right)}{g\left(u_{f}^{*}, v_{f}^{*}\right)}=-\frac{v_{f}^{*} \frac{\partial d_{2}}{\partial v}\left(u_{f}^{*}, v_{f}^{*}\right)}{d_{2}\left(u_{f}^{*}, v_{f}^{*}\right)}
\end{array}\right.
$$

and since

$$
\frac{\frac{\partial d_{1}}{\partial u}(u, v)}{d_{1}(u, v)}=\frac{\frac{\partial d_{2}}{\partial u}(u, v)}{d_{2}(u, v)}
$$

it follows that at a pair $\left(u_{f}^{*}, v_{f}^{*}\right)$ of optimizers, the partial elasticities of the payoff function $g$ equal the negative of the partial elasticities of the discount factors:

$$
\left\{\begin{array}{l}
\mathbb{E}_{\mathbb{L}_{u}}[g]\left(u_{f}^{*}, v_{f}^{*}\right)=-\mathbb{E}_{\mathbb{L}_{u}}\left[d_{1}\right]\left(u_{f}^{*}, v_{f}^{*}\right) \\
\mathbb{E} \mathbb{L}_{v}[g]\left(u_{f}^{*}, v_{f}^{*}\right)=-\mathbb{E L}_{v}\left[d_{2}\right]\left(u_{f}^{*}, v_{f}^{*}\right)
\end{array}\right.
$$

Notice that the payoff function $g$ is valued in currency at the time of harvesting, not its present-value.

\subsection{Example}

The illustration of the Faustmann solution uses the same model formulation as for the Wicksell problem. Recall the growth process $X$ satisfies (1) with $\mu(x, y)=\bar{\mu}\left(1-\gamma_{y} x\right)$ and $\sigma(x, y)=\bar{\sigma} \sqrt{x}$, where $\bar{\mu}, \bar{\sigma}$ and $\gamma_{y}$ are fixed positive constants, while each thinning decision produces a value of $X\left(\theta_{k}\right)=x_{\text {thin }}$ and harvesting restarts the forest value at $X\left(\eta_{k}\right)=x_{n e w}$. Also recall that the differential operators for the mean-reverting model are $A^{(y)} f(x)=\bar{\mu}\left(1-\gamma_{y} x\right) f^{\prime}(x)+\left(\bar{\sigma}^{2} / 2\right) x f^{\prime \prime}(x), y=1,2$, the increasing solutions of the eigenfunction equation (5) are given by (18) and the payoff functions are as in (19).

Table 2 displays the optimal thinning and harvesting levels $u_{f}^{*}$ and $v_{f}^{*}$ along with the optimal payoff for the Faustmann problem. Comparing these values with Table 1, one observes the effect of optimizing over infinitely many cycles reduces both the optimal thinning and harvesting levels while at the same time increases the payoff received. Moreover, these optimal thinning and harvesting levels are in agreement with the comments by Morrow (1981) on the need to thin a stand of hardwoods when dbh is in the 4 to 10 inch range and the valuation of the trees is high when dbh is 24 to 28 inches $(\sim 60 \mathrm{~cm}$ to $\sim 70 \mathrm{~cm})$.

$\begin{array}{lccc}x_{\text {thin }} & u_{f}^{*} & v_{f}^{*} & V^{f}\left(x_{\text {new }}, 1\right) \\ 10 . & 28.5 & 60.7 & 3.405 \\ 12.5 & 27.0 & 60.7 & 3.677 \\ 15 . & 25.3 & 60.6 & 4.002 \\ 17.5 & 23.5 & 60.4 & 4.396 \\ 20 . & 21.4 & 60.3 & 4.879 \\ 22.5 & 19.0 & 60.1 & 5.481 \\ 25 . & 16.1 & 59.9 & 6.249\end{array}$

Table 2: Optimal thinning levels $u_{f}^{*}$ and harvesting levels $v_{f}^{*}$ (in $\mathrm{cm}$ ) for the Faustmann-Model as well as values of $V^{f}\left(x_{n e w}, 1\right)$ for various $x_{\text {thin }}$ values (in $\mathrm{cm}$ ) using two mean reverting processes; $\mu_{1}=\mu_{2}=1, \sigma_{1}^{2}=\sigma_{2}^{2}=0.03 ; 1 / \gamma_{1}=100,1 / \gamma_{2}=120$, $\alpha=0.03, x_{\text {new }}=0.5, \delta_{1}=0.7345, \varrho_{1}=0, \mathfrak{z}_{1}=0, c_{1}=9.1748, \delta_{2}=1.8254, \varrho_{2}=0.04502, \mathfrak{z}_{2}=56.6523, c_{2}=4.3862$. 


\section{Concluding Remarks}

This paper has examined a variation of the Wicksell single cycle and Faustmann on-going harvest rotation problems that includes thinning of the forest to promote better growth dynamics. The model adopts a deterministic pricing function. This choice can be understood to be derived from aggregate data over a long-term horizon and thus the problem under consideration treats rotation management as a long-term investment. One might use this problem to determine an approximate age or size of tree at which harvesting would occur. Then when one approaches the harvesting and marketing of the forest, one would take a short-term view and use a stochastic pricing model to decide on the precise harvesting time.

The analysis in this paper replaces the stochastic model by an infinite-dimensional linear program over a space of (deterministic) measures. This approach proved to be quite tractable for the problems under study in this paper. An interesting feature of this analysis is the reduction of the Faustmann infinite-cycle problem to a Wicksell single-cycle problem through the use of the strong Markov property for the growth process.

The results for the thinning-and-harvest problems immediately reduce to the known results for the harvest only problems. When there is no change in dynamics allowed, there is only one strictly increasing solution to the eigenvalue problem $A f=\alpha f$ and there would be no dependence on a $Y$ process. For the Wicksell problem, one eliminates the $g_{2}$ term from the optimization problem (12) since this would provide a second harvest opportunity. Theorem 3 then reduces to the known solution (see, e.g., Sødal (2002)). The reduction for the Faustmann problem is even more immediate. When harvesting is the only decision to make, there would be a single payoff function $g$, only one reinitializing point $x_{n e w}$, one decision level $u^{*}$ and as above only one increasing function $\psi$. The expression (22) in Theorem 5 then simplifies to the known result.

This methodology can be easily adapted to include additional features to the model. For instance, the owner of the forest stand may receive a running payment stream that depends on the size of the forest; such payments might represent the amenity value of the forest or carbon credit payments that are received as long as the forest is allowed to grow (see Helmes and Stockbridge (2010) for a single-cycle example using this methodology). The dynamics may also include sudden destruction due to fire or pests. Assuming the occurrences of such destruction are modelled by a Poisson process with some probability distribution on the size of the forest following the occurrence, the methods of this paper apply with only a minor modification to the generator $A$ and an adjustment to the discount factor $\alpha$.

\section{Acknowledgement}

The authors would like to thank Hans Volkmer for the simple proof of the optimization result in Proposition 1 .

\section{Appendix A. Optimization of Linear Program (11)}

In this appendix, we prove that an optimal choice of measures for (11) will place point masses on locations which maximize (12). To do so, however, we phrase the problem more generally as one of finding finite measures $\nu_{1}$ and $\nu_{2}$ so as to solve the linear programming problem

$$
\begin{cases}\text { Maximize } & \int g_{1}(x) \nu_{1}(d x)+\int g_{2}(y) \nu_{2}(d y) \\ \text { Subject to } & \int F_{1}(x) \nu_{1}(d x)+\int F_{2}(y) \nu_{2}(d y)=1 \\ & \int G_{1}(x) \nu_{1}(d x)-\int G_{2}(y) \nu_{2}(d y)=0\end{cases}
$$

in which $F_{i}$ are non-negative, measurable, $G_{i}$ are positive, measurable and $g_{i}$ are measurable functions on measurable spaces $\left(E_{i}, \mathcal{F}_{i}\right), i=1,2$. To be feasible, the measures $\nu_{1}$ and $\nu_{2}$ must be such that for $i=1,2$, $F_{i}$ and $G_{i}$ are integrable with respect to $\nu_{i}$. 
Proposition 1. Define the functions $H(x, y)=F_{1}(x) G_{2}(y)+G_{1}(x) F_{2}(y)$ and $h(x, y)=g_{1}(x) G_{2}(y)+$ $G_{1}(x) g_{2}(y)$ and assume $H$ is strictly positive. Then an upper bound on the optimal value of (A.1) is given by

$$
\int g_{1}(x) \nu_{1}(d x)+\int g_{2}(y) \nu_{2}(d y) \leq \sup _{(x, y)} \frac{h(x, y)}{H(x, y)}
$$

and if the function $h / H$ has a global maximum, then this bound is achieved.

Proof. Let $\left(\nu_{1}, \nu_{2}\right)$ be a feasible pair of measures and note that at least one (hence both) measures have positive mass. Let $\nu=\nu_{1} \times \nu_{2}$ be the product measure on $\left(E_{1} \times E_{2}, \mathcal{F}_{1} \times \mathcal{F}_{2}\right)$. Observe that

$$
\begin{aligned}
\int H d \nu & =\int F_{1} d \nu_{1} \int G_{2} d \nu_{2}+\int G_{1} d \nu_{1} \int F_{2} d \nu_{2} \\
& =\left(\int F_{1} d \nu_{1}+\int F_{2} d \nu_{2}\right) \int G_{2} d \nu_{2}
\end{aligned}
$$

where the second constraint has been used and hence

$$
\int \frac{H}{\int G_{2} d \nu_{2}} d \nu=1
$$

Thus a probability measure $\tilde{\nu}$ can be defined by taking $H /\left(\int G_{2} d \nu_{2}\right)$ to be the density with respect to $\nu$.

Now observe that

$$
\begin{aligned}
\int h d \nu & =\int g_{1} d \nu_{1} \int G_{2} d \nu_{2}+\int G_{1} d \nu_{1} \int g_{2} d \nu_{2} \\
& =\left(\int g_{1} d \nu_{1}+\int g_{2} d \nu_{2}\right) \int G_{2} d \nu_{2} .
\end{aligned}
$$

So as a result,

$$
\int g_{1} d \nu_{1}+\int g_{2} d \nu_{2}=\int \frac{h}{\int G_{2} d \nu_{2}} d \nu=\int \frac{h}{H} \cdot \frac{H}{\int G_{2} d \nu_{2}} d \nu=\int \frac{h}{H} d \tilde{\nu}
$$

For any probability measure $\tilde{\nu}$, the value of the objective is bounded above by $\sup _{x, y} \frac{h(x, y)}{H(x, y)}$. Moreover, when the ratio $h / H$ achieves its maximum, say at $\left(x^{*}, y^{*}\right)$, an optimal pair of measures $\left(\nu_{1}^{*}, \nu_{2}^{*}\right)$ can be determined by taking $\tilde{\nu}=\delta_{\left\{\left(x^{*}, y^{*}\right)\right\}}$. This then implies that the corresponding product measure $\nu^{*}$ also places a point mass on $\left\{\left(x^{*}, y^{*}\right)\right\}$ having mass $\frac{\int G_{2} d \nu_{2}}{H\left(x^{*}, y^{*}\right)}$ and moreover that the measure $\nu_{1}^{*}$ is a point mass on $\left\{x^{*}\right\}$ and similarly $\nu_{2}^{*}$ is a point mass on $\left\{y^{*}\right\}$. Now utilizing the two constraints of the linear program, we are able to determine the masses of $\nu_{1}^{*}$ and $\nu_{2}^{*}$ from the system of equations

$$
\begin{aligned}
F_{1}\left(x^{*}\right) \nu_{1}^{*}\left\{x^{*}\right\}+F_{2}\left(y^{*}\right) \nu_{2}^{*}\left\{y^{*}\right\} & =1 \\
G_{1}\left(x^{*}\right) \nu_{1}^{*}\left\{x^{*}\right\}-G_{2}\left(y^{*}\right) \nu_{2}^{*}\left\{y^{*}\right\} & =0
\end{aligned}
$$

yielding

$$
\nu_{1}^{*}\left\{x^{*}\right\}=\frac{G_{2}\left(y^{*}\right)}{F_{1}\left(x^{*}\right) G_{2}\left(y^{*}\right)+G_{1}\left(x^{*}\right) F_{2}\left(y^{*}\right)}, \quad \nu_{2}^{*}\left\{y^{*}\right\}=\frac{G_{1}\left(x^{*}\right)}{F_{1}\left(x^{*}\right) G_{2}\left(y^{*}\right)+G_{1}\left(x^{*}\right) F_{2}\left(y^{*}\right)} .
$$




$\begin{array}{cc}\begin{array}{c}\text { dbh } \\ \text { (in cm) }\end{array} & \begin{array}{c}\text { Tree Value Conversion Standards } \\ \text { (TVCS) }\end{array} \\ 25 & 0 \\ 30 & 1 \\ 35 & 3 \\ 40 & 8 \\ 45 & 17 \\ 50 & 29 \\ 60 & 58 \\ 70 & 94\end{array}$

Table B.3: Typical Change in Size and Value with Growth of a Sugar Maple

\section{Appendix B. Payoff Function $g_{2}$}

The form of the harvesting payoff function $g_{2}(x)=x \delta_{2} \cdot \frac{1+\tanh \left(\varrho_{2}\left(x-\mathfrak{z}_{2}\right)\right)}{2}-c_{2}$ is chosen since the parameters $\delta_{2}, \varrho_{2}, \mathfrak{z}_{2}$ and $c_{2}$ provide enough flexibility to accurately capture pricing data. For example, Morrow (1981) reports pricing data (based on 1972 prices) over their growth cycle for a number of different varieties of trees. Table B.3 displays the data for the growth of a sugar maple; dbh stands for the diameter at breast height. Tree Value Conversion Standards (TVCS) provide a measure of a tree's worth, based on the comparative value of the quantity and quality of expected yield of one-inch lumber, taking into account conversion costs such as harvesting, transporting, and milling. It is a standard by which trees of different sizes can be compared, but it excludes most price effects of inflation and the marketplace. A fit of this pricing data using the parameters $\delta_{2}=1.8254, \varrho_{2}=0.04502, \mathfrak{z}_{2}=56.6523$ and $c_{2}=4.3862$ is displayed in Figure B.1. We can see this family of pricing functions provides an excellent fit to the data; very good fits are also provided with an appropriate change in the parameters for the prices corresponding to other varieties of trees.

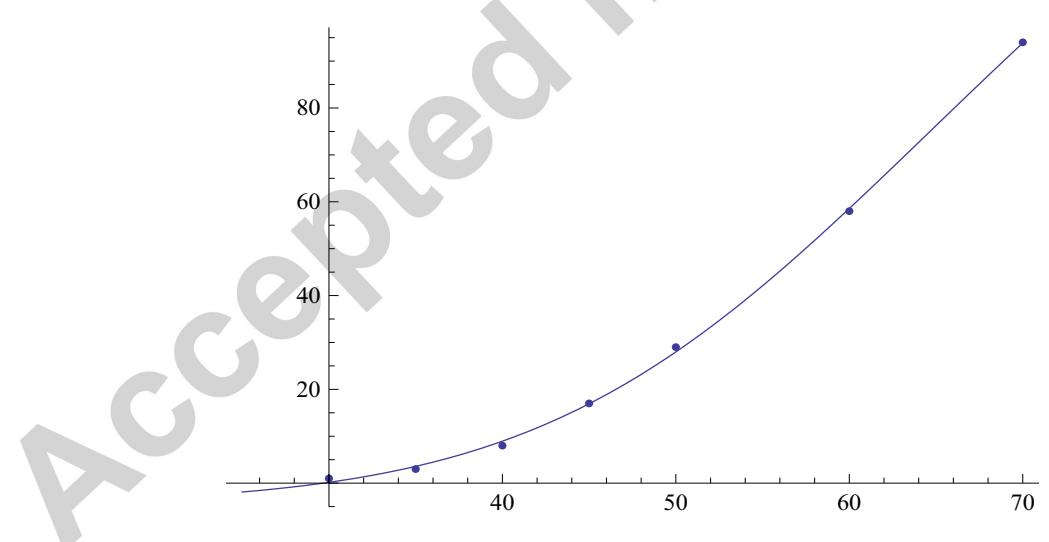

Figure B.1: Fit of $g_{2}$ to (dbh,TVCS) data in Table B.3.

\section{Appendix C. Expected Discount Factors}

Consider the thinning-and-harvesting rule whereby a dense forest is thinned whenever it reaches level $u$ and is harvested whenever a thinned forest achieves size $v$. Recall, the initial state of the forest is $(X(0), Y(0))=\left(x_{n e w}, 1\right)$ indicating that the stand is dense and new. Define the "zeroeth" harvesting time $\eta_{0}=0$. Now define the successive thinning and harvest times (for $k=1,2,3, \ldots$ ) by $\theta_{k}=\inf \left\{t \geq \eta_{k-1}\right.$ : 
$X(t-)=u\}$ and $\eta_{k}=\inf \left\{t \geq \theta_{k}: X(t-)=v\right\}$. We seek to determine the expressions for the expected discount factors

$$
E\left[\sum_{k=1}^{\infty} e^{-\alpha \theta_{k}}\right] \text { and } E\left[\sum_{k=1}^{\infty} e^{-\alpha \eta_{k}}\right] .
$$

To determine $E\left[e^{-\alpha \eta_{k}}\right]$ define $\tilde{\eta}_{k}=\eta_{k}-\theta_{k}$ and observe that $\tilde{\eta}_{k}$ gives the first hitting time of level $v$ of the process $X$ under the dynamics with $Y(t)=1$ starting at time $\theta_{k}$ in location $X\left(\theta_{k}\right)$ having distribution $\pi$ on $\left[x_{\min }, x_{\max }\right]$ independent of $\theta_{k}$. Recall from Remark 1, the Laplace transform of $\tilde{\eta}_{k}$ is given by $\left\langle\psi_{2}, \pi\right\rangle / \psi_{2}(v)$. Using the strong Markov property and subscripts on the expectation operator to indicate the initial value of $X$, we have

$$
\begin{aligned}
E_{x_{\text {new }}}\left[e^{-\alpha \eta_{k}}\right] & =E_{x_{\text {new }}}\left[e^{-\alpha\left[\theta_{k}+\left(\eta_{k}-\theta_{k}\right)\right]}\right] \\
& =E_{x_{\text {new }}}\left[e^{-\alpha \theta_{k}} E_{x_{\text {new }}}\left[e^{-\alpha\left(\eta_{k}-\theta_{k}\right)} \mid \mathcal{F}_{\theta_{k}}\right]\right] \\
& =E_{x_{\text {new }}}\left[e^{-\alpha \theta_{k}} E_{X\left(\theta_{k}\right)}\left[e^{-\alpha \tilde{\eta}_{k}}\right]\right] \\
& =E_{x_{\text {new }}}\left[e^{-\alpha \theta_{k}}\right] E\left[E_{X\left(\theta_{k}\right)}\left[e^{-\alpha \tilde{\eta}_{k}}\right]\right] \\
& =E_{x_{\text {new }}}\left[e^{-\alpha \theta_{k}}\right] \cdot \frac{\left\langle\psi_{2}, \pi\right\rangle}{\psi_{2}(v)}
\end{aligned}
$$

Similarly, let $\tilde{\theta}_{k}=\theta_{k}-\eta_{k-1}$, note $\tilde{\theta}_{k}$ is the first hitting time of $X$ (with $Y(t)=1$ ) started at time $\eta_{k-1}$ in location $x_{n e w}$ and $E\left[e^{-\alpha \tilde{\theta}_{k}}\right]=\psi_{1}\left(x_{n e w}\right) / \psi_{1}(u)$. Applying the strong Markov property, we have

$$
\begin{aligned}
E_{x_{\text {new }}}\left[e^{-\alpha \theta_{k}}\right] & =E_{x_{\text {new }}}\left[e^{-\alpha\left[\eta_{k-1}+\left(\theta_{k}-\eta_{k-1}\right)\right]}\right] \\
& =E_{x_{\text {new }}}\left[e^{-\alpha \eta_{k-1}} E_{x_{\text {new }}}\left[e^{-\alpha\left(\theta_{k}-\eta_{k-1}\right)} \mid \mathcal{F}_{\eta_{k-1}}\right]\right] \\
& =E_{x_{\text {new }}}\left[e^{-\alpha \eta_{k-1}} E_{X\left(\eta_{k-1}\right)}\left[e^{-\alpha \tilde{\theta}_{k}}\right]\right] \\
& =E_{x_{\text {new }}}\left[e^{-\alpha \eta_{k-1}}\right] \cdot \frac{\psi_{1}\left(x_{\text {new }}\right)}{\psi_{1}(u)} .
\end{aligned}
$$

Iterating (C.1) and (C.2) determines the summands of each series and hence yields

and

$$
\begin{aligned}
E\left[\sum_{k=1}^{\infty} e^{-\alpha \eta_{k}}\right]=\sum_{k=1}^{\infty}\left(\frac{\psi_{1}\left(x_{n e w}\right)}{\psi_{1}(u)} \cdot \frac{\left\langle\psi_{2}, \pi\right\rangle}{\psi_{2}(v)}\right)^{k} & =\frac{1}{1-\frac{\psi_{1}\left(x_{n e w}\right)\left\langle\psi_{2}, \pi\right\rangle}{\psi_{1}(u) \psi_{2}(v)}} \cdot \frac{\psi_{1}\left(x_{n e w}\right)\left\langle\psi_{2}, \pi\right\rangle}{\psi_{1}(u) \psi_{2}(v)} \\
& =\frac{\psi_{1}\left(x_{n e w}\right)\left\langle\psi_{2}, \pi\right\rangle}{\psi_{1}(u) \psi_{2}(v)-\psi_{1}\left(x_{n e w}\right)\left\langle\psi_{2}, \pi\right\rangle}
\end{aligned}
$$

$$
\begin{aligned}
E\left[\sum_{k=1}^{\infty} e^{-\alpha \theta_{k}}\right]=\sum_{k=1}^{\infty}\left(\frac{\psi_{1}\left(x_{n e w}\right)}{\psi_{1}(u)}\right)^{k} \cdot\left(\frac{\left\langle\psi_{2}, \pi\right\rangle}{\psi_{2}(v)}\right)^{k-1} & =\frac{\psi_{2}(v)}{\left\langle\psi_{2}, \pi\right\rangle} \sum_{k=1}^{\infty}\left(\frac{\psi_{1}\left(x_{n e w}\right)}{\psi_{1}(u)} \cdot \frac{\left\langle\psi_{2}, \pi\right\rangle}{\psi_{2}(v)}\right)^{k} \\
& =\frac{\psi_{1}\left(x_{n e w}\right) \psi_{2}(v)}{\psi_{1}(u) \psi_{2}(v)-\psi_{1}\left(x_{n e w}\right)\left\langle\psi_{2}, \pi\right\rangle} .
\end{aligned}
$$

\section{References}

Abramowitz, M., Stegun, I.A., 1965. Handbook of Mathematical Functions. Dover, New York.

Alvarez, L.H.R., Koskela, E., 2005. Wicksellian theory of forest rotation under interest rate variability. Journal of Economic Dynamics and Control 29, 529-545.

Baldursson, F.M., Karatzas, I., 1997. Irreversible investment and industry equilibrium. Finance and Stochastic 1, 69-89.

Borodin, A.N., Salminen, P., 2002. Handbook of Brownian Motion - Facts and Formulae. 2nd. ed., Birkhäuser, Basel. 
Brekke, K.A., Øksendal, B., 1994. Optimal Switching in an Economic Activity under Uncertainty. SIAM Journal on Control and Optimization 32, 1021-1036.

Brennan, M.J., Schwartz, E.S., 1985. Evaluating Natural Resource Investments. Journal of Business 58, $135-157$.

Buongiorno, J., 2001. Generalization of Faustmann's formula for stochatic forest growth and prices with Markov decision process models. Forest Science 47, 466-474.

Cho, M.J., Stockbridge, R.H., 2002. Linear programming formulation for optimal stopping problems. SIAM Journal on Control and Optimization 40, 1965-1982.

Clarke, H.R., Reed, W.J., 1989. The tree-cutting problem in a stochastic environment. Journal of Economic Dynamics and Control 13, 569-595.

Davies, K., 1996. Towards more accurate growth simulations and NPV appraisals: Using INFORM to project tree grade and market value increases. The Compiler 14, 18-23.

DeBald, P.S., Dale, M.E., 1991. Tree value conversion standards revisited. USDA Forest Service Research Paper NE-645.

Dixit, A., 1989. Entry and Exit Decisions under Uncertainty. Journal of Political Economy 97, 620-638.

Dixit, A., Pindyck, R.S., 1994. Investment under Uncertainty. Princeton University Press.

Duckworth, K., Zervos, M., 2001. A Model for Investment Decisions with Switching Costs. Annals of Applied Probability 11, 239-260.

Ethier, S.N., Kurtz, T.G., 1986. Markov Processes: Characterization and Convergence. Wiley, New York.

Faustmann, M., 1849. Berechnung des Werthes, welchen Waldboden, sowie noch nicht haubare Holzbestände für die Waldwirthschaft besitzen. Allgemeine Forst- und Jagdzeitung 15, 441-455.

Helmes, K., Röhl, S., Stockbridge, R.H., 2001. Computing moments of the exit time distribution for Markov processes by linear programming. Operations Research 49, 516-530.

Helmes, K., Stockbridge, R.H., 2003. Extension of Dale's moment conditions with application to the Wright-Fisher model. Stochastic Models 19, 255-267.

Helmes, K., Stockbridge, R.H., 2007. Linear Programming Approach to the Optimal Stopping of Singular Stochastic Processes. Stochastics 79, 309-335.

Helmes, K., Stockbridge, R.H., 2010. Construction of the value function and stopping rules in optimal stopping of onedimensional diffusions. Advances in Applied Probability 42, 158-182.

Itô, K., McKean, Jr., H.P., 1974. Diffusion Process and their Sample Paths. Springer-Verlag, New York.

Karlin, S., Taylor, H.M., 1981. A Second Course in Stochastic Processes. Academic Press, New York.

Kurtz, T.G., Stockbridge, R.H., 1998. Existence of Markov controls and characterization of optimal Markov controls. SIAM Journal on Control and Optimization 36, 609-653. Erratum: SIAM Journal on Control and Optimization 37 (1999), $1310-$ 1311.

Kurtz, T.G., Stockbridge, R.H., 2001. Stationary solutions and forward equations for controlled and singular martingale problems. Electronic Journal of Probability 6, Paper No. 14, 1-52.

Lumley, R.R., Zervos, M., 2001. A Model for Investments in the Natural Resource Industry with Switching Costs. Mathematics of Operations Research 26, 637-653.

Mendel, J.J., DeBald, P.S. and Dale, M.E., 1976. Tree value conversions standards for hardwood sawtimber. USDA Forest Service Research Paper NE-337.

Miller, R.A., Voltaire, K., 1983. A stochastic analysis of the tree paradigm. Journal of Economic Dynamics and Control 6, 371-386.

Morrow, R.R., 1981. Tree value: A basis for woodland management. Cornell University Department of Natural Resources Conservation Circular 19, 101-104.

Newman, D.H., 2002. Forestry's golden rule and the development of the optimal forest rotation literature. Journal of Forest Economics 8, 5-27.

Nordstrøm, C.J., 1975. A stochastic model for the growth period decision in forestry. Swedish Journal of Economics $77,329-337$.

Penttinen, M.J., 2006. Impact of stochastic price and growth processes on optimal rotation age. European Journal of Forest Research 125, 335-343.

Sødal, S., 2002, The stochastic rotation problem: A comment. Journal of Economic Dynamics and Control 26, $509-515$.

Willassen, Y., 1998. The stochastic rotation problem: A generalization of Faustmann's formula to stochastic forest growth. Journal of Economic Dynamics and Control 22, 573-596.

Zervos, M., 2002. A Problem of Sequential Entry and Exit Problems Combined with Optimal Stopping. SIAM Journal on Control and Optimization 42, 397-421. 